\title{
Influence of heterogeneous freezing on the microphysical and radiative properties of orographic cirrus clouds
}

\author{
H. Joos ${ }^{1}$, P. Spichtinger ${ }^{2}$, P. Reutter ${ }^{2}$, and F. Fusina ${ }^{1, *}$ \\ ${ }^{1}$ Institute for Atmospheric and Climate Science, ETH Zurich, Zurich, Switzerland \\ ${ }^{2}$ Institute for Atmospheric Physics, Johannes Gutenberg University Mainz, Mainz, Germany \\ * now at: Swiss International Airlines, Zurich, Switzerland \\ Correspondence to: H. Joos (hanna.joos@env.ethz.ch)
}

Received: 23 May 2013 - Published in Atmos. Chem. Phys. Discuss.: 6 July 2013

Revised: 10 April 2014 - Accepted: 27 May 2014 - Published: 4 July 2014

\begin{abstract}
The influence of heterogeneous freezing on the microphysical and optical properties of orographic cirrus clouds has been simulated with the large eddy simulation model EULAG. Idealised simulations with different concentrations of ice nuclei (IN) in a dynamically dominated regime with high vertical velocities have been performed. Furthermore the temperature at cloud formation as well as the critical supersaturation for initiation of heterogenous freezing have been varied. The shortwave, longwave and net cloud forcing has been calculated under the assumption that the clouds form between 06:00 and 12:00 local time (LT) or between 12:00 and 18:00 LT. In general it can be seen that the onset of homogeneous freezing is shifted in time depending on the IN concentration, as part of the available water vapour is depleted before the critical threshold for homogeneous freezing is reached. Although the high vertical velocities in an orographic gravity wave lead to a strong adiabatic cooling followed by high ice supersaturations, even a small number concentration of IN of the order of $5 \mathrm{~L}^{-1}$ is able to strongly decrease the simulated ice crystal number burden (ICNB), ice water path (IWP) and optical depth of the cloud. In general, the ICNB, IWP and optical depth strongly decrease when the IN concentrations are increased from 0 to $50 \mathrm{~L}^{-1}$. The absolute values of the shortwave, longwave and net cloud forcing are also reduced with increasing IN concentrations. A cloud will produce a net warming or cooling depending on the IN concentration, the temperature and the time of day when the cloud forms. The clouds that form between 06:00 and 12:00 LT are mainly cooling, whereas the clouds with the same microphysical properties can lead to a warming when they form between 12:00 and 18:00 LT. In
\end{abstract}

order to predict the radiative forcing of cirrus clouds it is therefore necessary to take the correct dynamical and thermodynamical processes as well as the possible existence and freezing threshold of heterogeneous IN into account, not only for low vertical velocities but also for dynamically dominated regimes like orographic cirrus.

\section{Introduction}

Cirrus clouds, i.e. high clouds consisting exclusively of ice crystals, play a crucial role in the climate system and cover approximately $30 \%$ of the Earth (Wylie and Menzel, 1999). Ice crystals interact with the short- and longwave radiation and have great potential to modulate the Earth's radiative budget. Depending on their microphysical and optical properties, cirrus clouds can either cool or warm the Earthatmosphere system. On the one hand, ice crystals scatter the shortwave radiation back into space, thus leading to a cooling (albedo effect). On the other hand, the longwave radiation can be trapped efficiently, leading to a warming (greenhouse effect). In general, optically thin cirrus clouds exert a warming as the absorption of infrared radiation and re-emission at lower temperatures dominates the scattering of incoming solar radiation, whereas optically thick cirrus clouds lead to a cooling due to the scattering of shortwave radiation back into space being the dominant process. Whether cirrus clouds lead to a net warming or cooling thus depends on their macrophysical properties such as the optical thickness of the cloud; these properties are determined by microphysical properties such as the ice crystal number concentration, 
ice water content, crystal shape and size (Wendisch et al., 2007). The microphysical properties of a cirrus cloud depend, on the one hand, on the underlying dynamics and, on the other hand, on the freezing mechanism. Cirrus clouds can form by homogeneous and heterogeneous freezing. Homogeneous freezing means the freezing of supercooled solution droplets. Solution droplets of a certain size start to freeze if the nucleation rate is so large that the freezing probability approaches 1 . The nucleation rate depends only on temperature and relative humidity and is mostly independent of the nature of the solute (Koop et al., 2000). Heterogeneous freezing denotes a freezing process which is associated with solid aerosol particles, the so-called ice nuclei (IN). The supersaturation threshold which is needed for the initiation of heterogeneous freezing depends on the properties of the IN. Whereas for homogeneous freezing very high supersaturations in excess of $45 \%$ are needed (Koop et al., 2000), heterogeneous freezing can be initiated at much lower supersaturations (DeMott et al., 2003; Möhler et al., 2006). It has been shown in different studies that the existence of IN and the occurrence of heterogeneous freezing can alter the formation of cirrus clouds significantly (Sassen and Benson, 2000; Khvorostyanov and Curry, 2005; DeMott et al., 1997). Spichtinger and Cziczo (2010) investigated in a box model study the influence of IN on the homogeneous freezing at relatively low updraft speeds $\left(0.05-0.2 \mathrm{~ms}^{-1}\right)$. They showed that the existence of IN that freeze at a critical supersaturation lower than for homogeneous freezing strongly modifies the subsequent homogeneous freezing and leads to a strong reduction in the simulated ice crystal number concentration (ICNC). The depletion of water vapour by the heterogeneously frozen ice crystals delays the onset and weakens the homogeneous freezing event. The effect of a reduced ICNC due to a previous heterogeneous freezing event compared to a purely homogeneous freezing event is also called the "negative Twomey effect" (Kärcher and Lohmann, 2003; Kärcher et al., 2007). Spichtinger and Gierens (2009c) investigated the effect of heterogeneous freezing with the cloudresolving model EULAG. They were able to show that low IN number concentrations of the order of $5 \mathrm{~L}^{-1}$ modify the microphysical properties of the cloud, at least for low updraft speeds of the order of $0.04-0.08 \mathrm{~m} \mathrm{~s}^{-1}$. In contrast to previous studies, we investigate the influence of IN on the clouds microphysical and optical properties in a highly dynamically dominated regime with vertical velocities of up to $0.8 \mathrm{~m} \mathrm{~s}^{-1}$ in an orographic gravity wave. Dean et al. (2005) and Joos et al. $(2008,2010)$ showed that orographically induced cirrus clouds contributes considerably to the cirrus cloud amount over and in the lee of mountains and are therefore also important for the radiative budget of the Earth. Additionally, we investigate how the changed microphysical properties modify the radiative properties of the clouds. Fusina et al. (2007) showed that the ice crystal number concentration plays an important role in the transition from the warming to the cooling regime. As the existence of IN has the potential to strongly modify the ICNC, the occurrence of heterogeneous freezing has to be taken into account for the correct calculation of the cirrus' optical properties together with the correct representation of the vertical velocity, which also strongly determines the ICNC (Kärcher and Lohmann, 2002; Spichtinger and Gierens, 2009b). It was previously thought that cirrus clouds lead to a net global warming (Chen et al., 2000). However, a significant part of the uncertainties in climate predictions arises from the representation of cirrus clouds in the climate models (Zhang et al., 2005). This is caused by the complex interaction of thermodynamical and dynamical processes which are not included correctly in climate models or not resolved by the coarse grid. The poor representation of cirrus clouds and their microphysical and optical properties in global climate models makes it especially difficult to estimate the changes in a future climate and exhibits a major uncertainty for predicting the future climate. In Joos et al. (2009) a first estimate of changes in the microphysical properties of orographic cirrus in a future climate is presented based on large eddy simulations (LES) with the EULAG model, whereas in Joos et al. (2010) changes in the microphysical and radiative properties are calculated with the global climate model ECHAM5. However, in both studies only homogeneous freezing was considered. In order to gain insight into the complex interactions between dynamics, microphysics and radiation, we therefore present the results of 38 radiation calculations of orographic cirrus clouds. To understand the important contributors to sub-grid processes related to the interaction between dynamics, microphysics and radiation, we investigate the role of nucleation mechanism, ice nuclei concentration, temperature and diurnal cycle in modulating the simulation of cirrus microphysical and radiative properties. The orographic cirrus clouds and its microphysical properties have been simulated with the LES model EULAG (Prusa et al., 2008), which contains detailed ice microphysics (Spichtinger and Gierens, 2009a). The results of the microphysical properties are then passed as input into a two-stream radiative transfer code (Fu and Liou, 1993); in other words, an offline calculation of radiative effects is performed. This model chain allows us to determine the influence of heterogeneous freezing on the microphysical and radiative properties of orographic cirrus clouds. The paper is organised as follows: in Sect. 2.1 the EULAG model and the radiative transfer code are introduced briefly. In Sect. 2.2 and Sect. 2.3, the simulation of the microphysical properties with EULAG and the calculation of the radiative properties are explained, respectively. Section 3.1 shows a detailed analysis of a simulation with homogeneous freezing only and a simulation where $10 \mathrm{IN} \mathrm{L}^{-1}$ are added. The results of all simulations are explained in Sect. 3.2, and we summarise our work in Sect. 4. 


\section{Model simulations}

\subsection{Model description}

For this study we use the anelastic, non-hydrostatic model EULAG (Prusa et al., 2008) for simulating the orographic cirrus clouds. As detailed ice microphysics are implemented in this model (Spichtinger and Gierens, 2009a), ice crystal number concentration as well as ice water content are prognostic variables. In our simulations, homogeneous and heterogeneous freezing is considered. The homogeneous nucleation rate is parameterised according to Koop et al. (2000) and the background sulfuric acid aerosols are distributed log-normally with an aerosol number concentration of $N_{\mathrm{a}}=200 \mathrm{~cm}^{-3}$, a geometric standard deviation of $\sigma_{r}=1.4$ and a modal radius of $r_{\mathrm{m}}=25 \mathrm{~nm}$. For heterogeneous freezing a very simple parameterisation is used. The number of available heterogeneous ice nuclei (IN) is prescribed and it is assumed that all IN become ice crystals at a prescribed critical relative humidity with respect to ice $\left(\mathrm{RHi}_{\text {het }}\right)$. If the ice crystals evaporate, the IN can be released back into the atmosphere. The use of such a simple heterogeneous freezing parameterisation like in this study is justified as it is not within the scope of this paper to investigate different freezing mechanisms. We want to investigate how the microphysical and optical properties of the cloud change if there were a certain number of IN that freeze at a given $\mathrm{RHi}$, no matter which exact freezing mechanism is active. For a detailed description and discussion of the scheme, see Spichtinger and Gierens (2009a). For the offline calculation of the radiative properties, a two-stream radiative transfer code is used. It contains 6 bands in the solar and 12 bands in the thermal infrared regime (Fu and Liou, 1993). The required microphysical variables are ice water content (IWC) and ICNC. Based on these variables the effective ice crystal size is calculated. Ice crystals are assumed to be small hexagonal columns and their effective ice crystal size is calculated under the assumption of randomly oriented columns (Fusina et al., 2007; Ebert and Curry, 1992). For the treatment of many arbitrary classes of ice, a generalised effective size for the ice crystal size distributions is derived. The derivation is described in detail in Appendix B. Furthermore, the solar zenith angle is calculated corresponding to the time of day, the day of year and the geographical latitude; the solar surface albedo is set to 0.3 , representative of mid-latitude conditions, the infrared surface emissivity is assumed to be 1 and the solar constant is $1340 \mathrm{Wm}^{-2}$.

\subsection{Simulation of microphysical properties}

In order to calculate orographic cirrus clouds and their microphysical properties we use a set of 18 idealised LES simulations. The setup consists of a 2-D domain ( $x-z$ plane) with horizontal extension of $320 \mathrm{~km}$ and vertical extension of $20 \mathrm{~km}$ with a bell-shaped mountain in the middle of the domain with a height of $600 \mathrm{~m}$ and a half-width of $10 \mathrm{~km}$. The horizontal resolution is $250 \mathrm{~m}$ and the vertical resolution is $50 \mathrm{~m}$. The model is initialised with the ambient potential temperature $\theta(z)$ and pressure $p(z)$ profiles according to Clark and Farley (1984), using a constant Brunt-Väisäla frequency over the whole troposphere. The tropopause is set at $z_{\mathrm{TP}}=13 \mathrm{~km}$, followed by higher stratification in the upper part of the domain $(13 \mathrm{~km} \leq z \leq 20 \mathrm{~km})$. Additionally, a wind profile $u(z)$ is prescribed. The wind speed increases between 0 and $2 \mathrm{~km}$ height from 4 to $9 \mathrm{~m} \mathrm{~s}^{-1}$. Above that level it stays constant $\left(u(z)=9 \mathrm{~m} \mathrm{~s}^{-1}\right)$ until a height of $12 \mathrm{~km}$, where it starts to decrease linearly until a height of $15 \mathrm{~km}$ to $-10 \mathrm{~m} \mathrm{~s}^{-1}$. The dynamical timestep is $\mathrm{d} t=2.5 \mathrm{~s}$ and the microphysical time step is $\mathrm{d} t_{\mathrm{m}}=\frac{\mathrm{d} t}{10}=0.25 \mathrm{~s}$. This setup leads to vertically propagating wave packets, forming a standing wave. Additionally, an ice-supersaturated region (ISSR) has been implemented at a height of 8500 to $9500 \mathrm{~m}$. For a more detailed description of the model setup, see also Sect. 4 of Joos et al. (2009). In this study it is also shown that the model represents the measurements of the ice crystal number concentration and ice water content of an orographic cirrus cloud, taken during the INCA campaign (Gayet et al., $2004)$ very well. The orographic cirrus cloud was measured on 5 April 2000 between 18:00 and 19:00 UTC on a flight track at $53^{\circ} \mathrm{S}$ over the Andes. The EULAG model was initialised with pressure, temperature and humidity fields for this date and a realistic topography for $53^{\circ} \mathrm{S}$ has been implemented. Since there is good agreement between measurements and model results, the proper use of the model for idealised orographic cirrus cloud studies is anticipated. Our simulations were run for $6 \mathrm{~h}$ with an output frequency of $10 \mathrm{~min}$. After approximately 90 min (depending on the number of IN) a cloud starts to form over the mountain and is present over the rest of the simulation time. In order to investigate the influence of heterogeneous freezing we performed one reference simulation with homogeneous freezing only and simulations where the heterogenous freezing is active as well. We run different setups in order to scan properly through the parameter space (temperature, RHi, IN concentration, nucleation threshold). In order to test the sensitivity to the number concentration of IN, simulations with 5,10 and $50 \mathrm{IN} \mathrm{L}^{-1}$ have been carried out. Furthermore, the temperature profile for initialising the model has been shifted such that the temperature inside the ISSR is $10 \mathrm{~K}$ warmer or colder then the reference case, respectively. The shift in the temperature profile does not change the flow regime as it is done in a way that the Brunt-Väisäla frequency stays the same as in the reference case. For the reference case, the temperature inside the ISSR at a height of $9000 \mathrm{~m}$ is $220.7 \mathrm{~K}$, for the cold profile $210.1 \mathrm{~K}$ and for the warm profile $229.9 \mathrm{~K}$. Additionally, the RHi which is required for initiating the heterogeneous freezing ( $\mathrm{RHi}_{\text {het }}$ ) is set to 120,130 and $140 \%$. However, the simulations with $\mathrm{RHi}_{\text {het }}=120$ and $140 \%$ are only performed for the reference temperature profile. In all simulations a steady mountain wave forms. The vertical velocities 

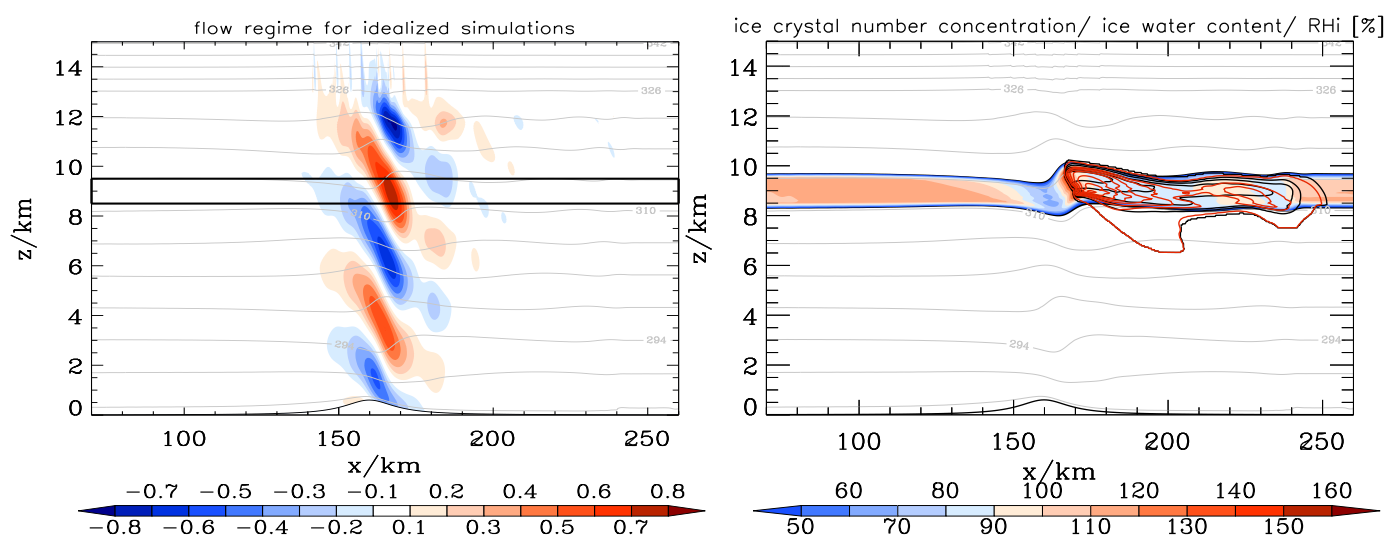

Figure 1. Flow regime after $5 \mathrm{~h}$ used for the idealised simulations HOM (left). Grey lines indicate lines of constant potential temperature with an increment of $4 \mathrm{~K}$. The black rectangle shows the position of the ISSR. Colours indicate the vertical velocities (left) and the relative

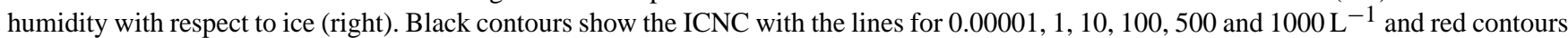
the IWC with lines for $0.0001,1,3,6,9,12,15$ and $18 \mathrm{mg} \mathrm{m}^{-3}$ for the simulation HOM. Taken from Joos et al. (2009).

reach values up to $0.8 \mathrm{~m} \mathrm{~s}^{-1}$ and the ISSR is located at a height where the updraft speed reaches its maximum. Thus, the clouds form in a dynamically dominated regime where the high updraft speeds lead to the formation of high ice supersaturations such that an orographic cirrus cloud can form. In Fig. 1 vertical velocities, relative humidity with respect to ice (RHi), ice crystal number concentration (ICNC) and the ice water content (IWC) are displayed for the simulation with homogeneous freezing only (HOM) for the reference temperature profile and $\mathrm{RHi}_{\text {het }}=130 \%$ after $5 \mathrm{~h}$. It can be seen that a cloud forms above the mountain crest that is advected $\sim 80 \mathrm{~km}$ downstream. If the crystals become large enough, they start to sediment and sublimate in the ice subsaturated air below the cloud. This effect can be seen from the red and black lines in Fig. 1, right between $\sim 170$ and $200 \mathrm{~km}$, where IWC and ICNC are present in subsaturated air. In Fig. 2, all LES simulations are summarised. For every temperature profile (reference, warm and cold), simulations with homogeneous freezing only and 5, 10 and $50 \mathrm{INL}^{-1}$ are performed (HOM, 5 IN, 10 IN, 50 IN), while it is assumed that heterogeneous freezing is initiated at $\mathrm{RHi}=130 \%$. This results in 12 simulations. Additionally, for the reference temperature profile only, the freezing threshold is shifted to 120 and $140 \%$ for the simulations $5 \mathrm{IN}, 10 \mathrm{IN}$ and $50 \mathrm{IN}$ ( 6 additional simulations). In total we therefore obtain 18 LES simulations which serve as input for the offline radiation calculation.

\subsection{Simulation of cirrus radiative properties}

The simulated ice crystal number concentration and ice water content obtained in the 18 LES simulations are used as an input for the radiative transfer code. In order to investigate the influence of the zenith angle on the results we performed radiation calculations from 06:00 to 12:00 LT as well as from 12:00 to 18:00 LT. The zenith angle is representative of $50^{\circ} \mathrm{N}$ and for 21 March. Radiation transfer calculations are carried

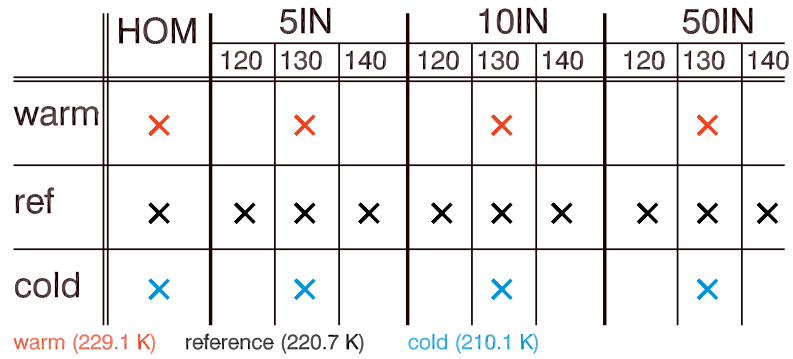

Figure 2. Summary of all 18 LES simulations. Each symbol represents one simulation. The temperature profile is shown in the vertical, whereas the temperature indicated at the bottom refers to the temperature inside the ISSR. The whole profiles can also be found in Joos et al. (2009). The horizontal shows how many IN are included in the simulation from 0 in $\mathrm{HOM}$ to $50 \mathrm{~L}^{-1}$ in the $50 \mathrm{IN}$ simulation. Furthermore, the critical relative humidity at which the IN are assumed to initiate heterogeneous freezing is shown, namely at 120,130 or $140 \%$.

out every $10 \mathrm{~min}$ with updated ice crystal number concentration and ice water content as well as actual zenith angle. The output of the radiative transfer code is given by a time series of cloud optical depth $\tau$ and radiative fluxes at the top of the atmosphere for the infrared as well as solar spectrum over $6 \mathrm{~h}$ for each simulation. Based on the radiative fluxes the longwave, shortwave and net cloud forcing is calculated. As the cold (warm) temperature profiles have a surface temperature of $270 \mathrm{~K}(298 \mathrm{~K})$, representing the conditions during the northern Hemispheric mid-latitude winter (summer), additional radiation calculations have been performed in which the solar zenith angle is calculated for the 21 December (21 June) instead of 21 March. For each of the 18 LES simulations, radiation calculations are performed for different local times and different seasons. We thus obtain 38 radiation calculations. Four calculations for each of the temperature 
profiles, namely with homogeneous freezing only and with 5,10 and $50 \mathrm{IN} \mathrm{L}^{-1}$, calculated for two different time slices of the day (06:00 to 12:00 and 12:00 to 18:00 LT) are performed. This leads to 24 radiation calculations. Additionally, 6 radiation calculations for the reference temperature profile with 5, 10 and $50 \mathrm{IN} \mathrm{L}^{-1}$ are calculated under the assumption that the IN freeze at a critical supersaturation of $\mathrm{RHi}_{\text {het }}=120$ or $140 \%$. Furthermore, eight simulations for homogeneous freezing only and 5, 10 and $50 \mathrm{IN} \mathrm{L}^{-1}$ for the cold (warm) temperature profiles have been performed in which the solar zenith angle is calculated for the 21 June (21 December). In Fig. 3 all 38 radiation calculations are summarised. Each symbol represents one radiation calculation and the colours of the symbols show for which temperature profile each radiation calculation is performed, while blue, black and red symbols refer to the cold, reference and warm profiles, respectively. Please note that for some radiation calculations the same LES simulations are used as an input. For example, the LES simulation $5 \mathrm{IN}$ with $\mathrm{RHi}_{\text {het }}=130 \%$ serves as an input for the radiation calculations from 06:00 to 12:00 and 12:00 to 18:00 LT. All radiation calculations in the first two rows which are illustrated either as star or rhombus and which have the same colour use the same LES simulation as input. In the following, a simulation where only homogenous freezing is considered as well as a simulation with $10 \mathrm{IN}$ for both 06:00 and 12:00 LT, the reference temperature profile and $\mathrm{RHi}_{\text {het }}=130 \%$ are explained in detail before a summary of all simulations is given in Sect. 3.2.

\section{Results}

In order to compare the different simulations, the vertically integrated IWC and ice water path (IWP) as well as the vertically integrated IWC, the so-called ice water path (IWP) as well as the vertically integrated ICNC, the so-called ice crystal number burden (ICNB) are calculated for every timestep (10 min). Furthermore, the optical depth $\tau$ as well as the longwave, shortwave and net cloud forcing are calculated for all simulations. The optical depth $\tau_{i}$ for a wavelength interval $i$ is calculated according to Fu and Liou (1993) and is given by

$\tau_{i}=\operatorname{IWP} \cdot\left(a+\frac{b}{D_{\mathrm{e}}}\right)$,

where IWP is the ice water path in $\mathrm{g} \mathrm{m}^{-3}, D_{\mathrm{e}}$ is the generalised size in micrometres, which can be calculated as described in Appendix B, and $a_{i}$ and $b_{i}$ are coefficients that depend on the wavelength interval. For IR emissivities, a similar relationship holds. However, one can conclude from coefficient analysis that the size is more important for solar radiation than for thermal radiation, which is strongly driven by ice mass. The optical depth $\tau$ is determined using the wavelength interval of visible light. The cloud forcing $(\mathrm{CF})$

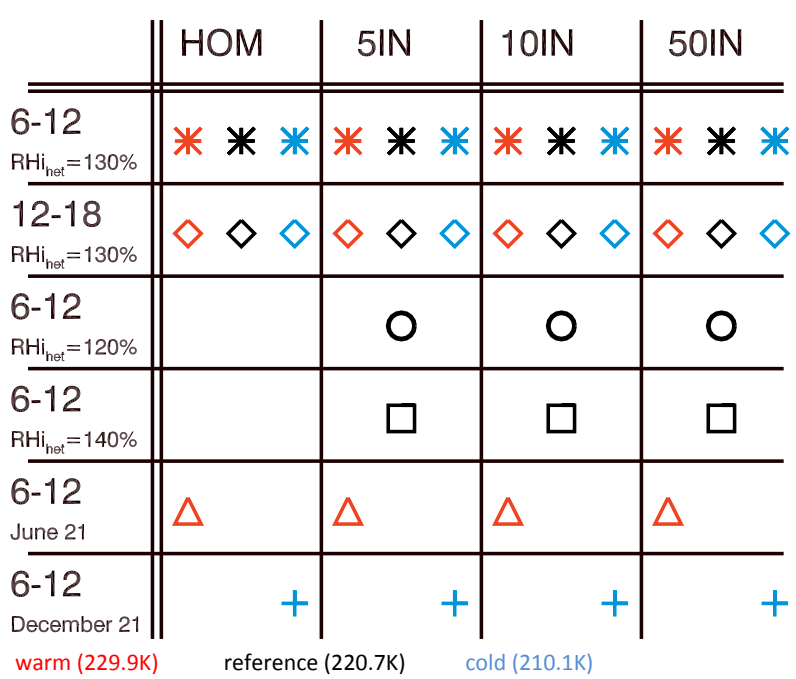

Figure 3. Summary of all 38 radiation calculations. Each symbol represents one radiation calculation. The horizontal shows how many IN are included in the simulation from 0 in $\mathrm{HOM}$ to $50 \mathrm{~L}^{-1}$ in simulation 50 IN. The vertical shows the time of day simulated; the colour of each symbol shows for which temperature profile the simulation is performed, with blue for the cold $(T=210.1 \mathrm{~K})$, black for the reference $(T=220.7 \mathrm{~K})$ and red for the warm $(T=229.9 \mathrm{~K})$ profile. These profiles can be found in Joos et al. (2009). Please note that the simulations with $\mathrm{RHi}_{\text {het }}=120 \%$ and $140 \%$ are only performed from 06:00 to 12:00 LT and for the reference temperature profile only (black symbols). The simulations for 21 June (21 December) are only done for 06:00-12:00 LT and the warm (cold) temperature profile. The symbols used in the table are the same as the ones in the figures in Sect. 3.2.

is defined as the difference between the clear-sky net top-ofatmosphere (TOA) radiation and the net TOA radiation with clouds. The $\mathrm{CF}$ can be calculated for the shortwave radiation such that $\mathrm{SCF}=S_{\mathrm{cs}}-S_{\text {cloud }}$, where $S_{\mathrm{cs}}$ is the shortwave clear-sky TOA radiation and $S_{\text {cloud }}$ is the shortwave TOA radiation with clouds. The same can be done for the longwave radiation, where $\mathrm{LCF}=L_{\mathrm{cs}}-L_{\text {cloud }}$ (Klein and Hartmann, 1993). SCF is negative as $S_{\text {cloud }}$ is larger than the corresponding clear-sky value as clouds scatter more radiation back into space than the underlying surface. LCF is positive as more longwave radiation is trapped when clouds are present. This effect is strongest for high, cold clouds as they radiate back into space with a much lower temperature than the underlying warmer surface. In order to compare the results for the 38 different radiation calculations, the scenario with pure homogeneous freezing and the reference temperature profile as well as the simulation with $10 \mathrm{IN}$ and $\mathrm{RHi}_{\text {het }}=130 \%$ for the time period 06:00 to 12:00 LT are described in more detail before a summary of all simulations is discussed in Sect. 3.2. 

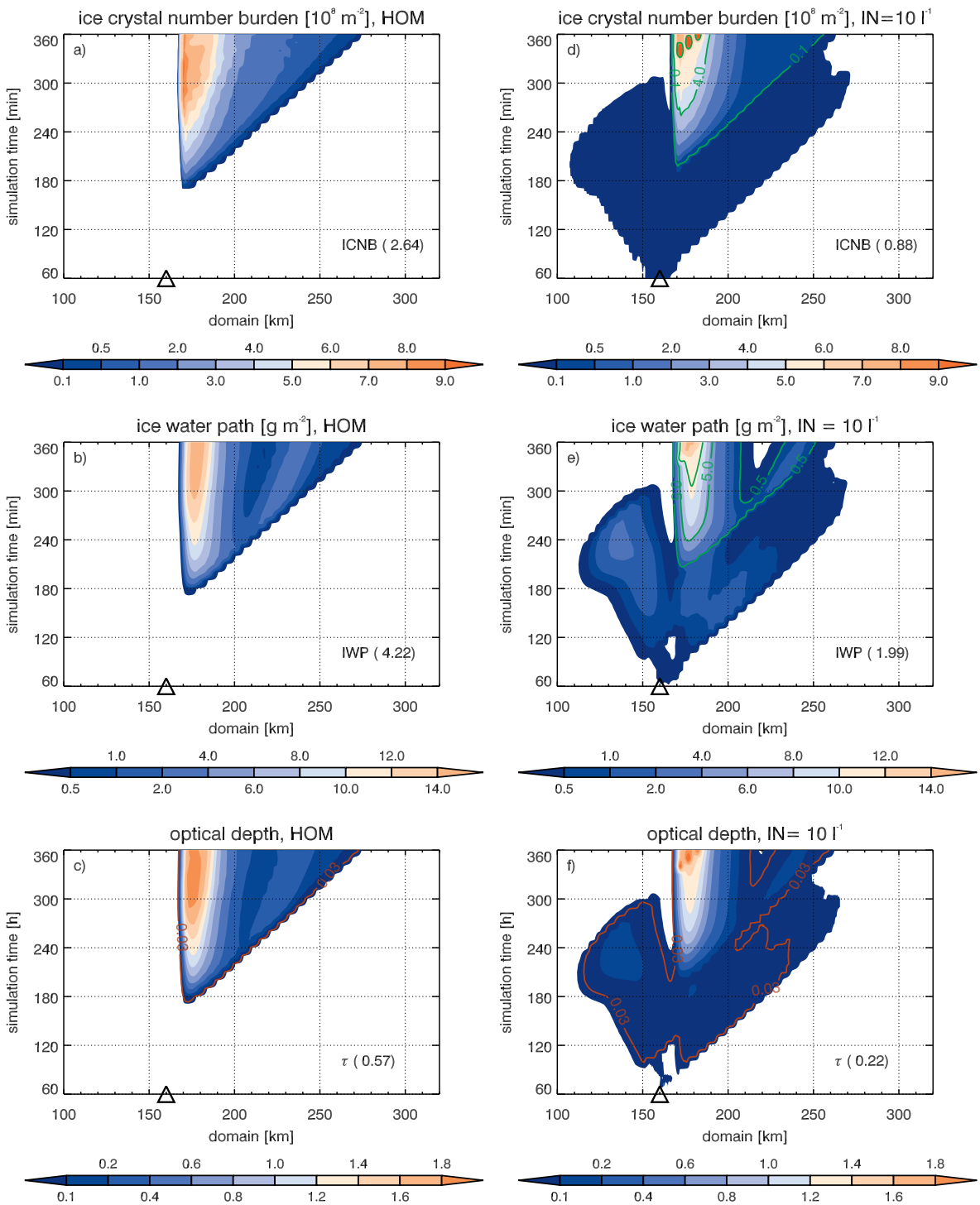

Figure 4. Time evolution of (a, d) ice crystal number burden, $(\mathbf{b}, \mathbf{e})$ ice water path and (c, f) optical depth for simulation HOM (left column) and $10 \mathrm{IN}$ (right column) for the reference temperature profile and $\mathrm{RHi}_{\text {het }}=130 \%$. The red line shows where $\tau=0.03$, and the triangle denotes the position of the top of the mountain. The green lines show the ICNB and IWP for the homogeneously frozen ice crystals with values of $0.1,4,9 \times 10^{8} \mathrm{~m}^{-2}$ for ICNB and $0.5,5,8 \mathrm{~g} \mathrm{~m}^{-2}$ for the IWP. The numbers in brackets show the mean values; the mean is only calculated where $\tau>0.03$. Note that the time of the day is not specified for these simulations without radiation.

\subsection{Detailed discussion of simulation HOM and 10 IN}

\subsubsection{Simulation HOM}

In order to compare the different simulations, the time evolutions of the microphysical properties IWP, ICNB and optical depth $\tau$ as well as the longwave (LCF), shortwave (SCF) and net cloud forcing (NCF) are shown. The left column of Fig. 4 shows the time evolution of IWP, ICNB and $\tau$ for the reference simulation where only homogeneous freezing is active (HOM) for the reference temperature profile. After $\sim 3 \mathrm{~h}$ the critical supersaturation for homogeneous freezing is reached and a cloud starts to form. The nucleation takes place close to the mountain crest, where the updraft speeds are highest. The ice crystals then grow due to deposition of water vapour and are partly advected downstream and sediment to lower levels. As time progresses, more crystals form as the existing ice crystals cannot deplete enough water vapour. Therefore the supersaturation with respect to ice stays high enough and new crystals can form until an equilibrium between the production of supersaturation due to the vertical velocities and the depletion of water vapour by the existing ice crystals is reached and the ICNB stays more or less constant. In connection with the nucleation of ice crystals, 

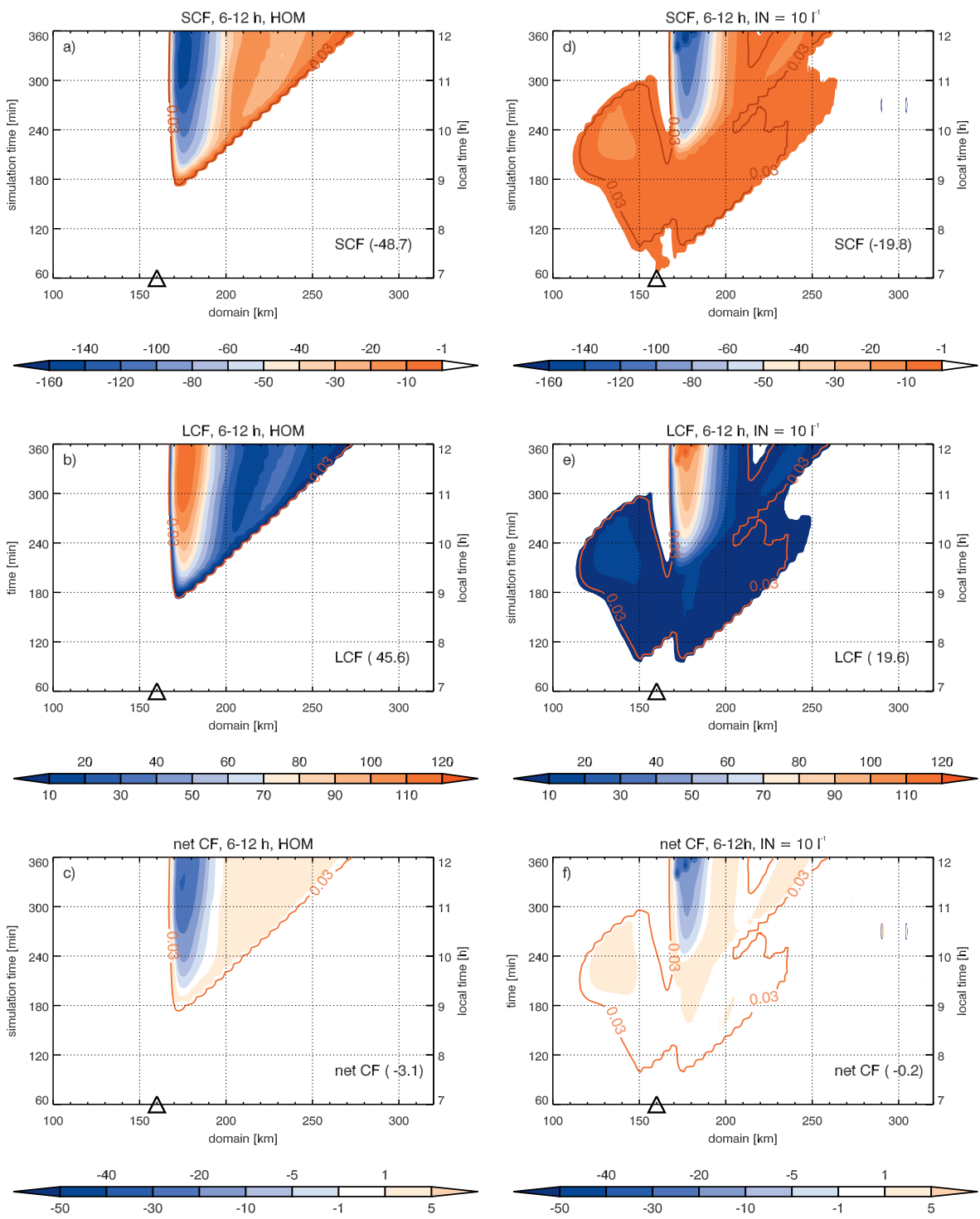

Figure 5. Time evolution of (a, d) shortwave cloud forcing (SCF), (b, e) longwave cloud forcing (LCF) and (c, f) net cloud forcing (NCF) for simulation HOM (left column) and $10 \mathrm{IN}$ (right column) for the reference temperature profile from 06:00 to 12:00 LT. The red line shows where $\tau=0.03$ and the triangle denotes the position of the top of the mountain. The numbers in brackets show the mean values; the mean is only calculated where $\tau>0.03$. Note that the time of the day is specified for these simulations with radiation.

the IWP increases with time. The highest values can be seen close to the mountain crest where the crystals form and grow. As the crystals start to sediment and sublimate during the downstream advection (see also Fig. 1, right panel), the IWP decreases with increasing distance from the formation region. At $x \sim 220 \mathrm{~km}$ a second small updraft region in the wave provides enough supersaturation such that the crystals grow again; however it is not high enough to reach the critical threshold for homogeneous nucleation again and no new crystals form. The corresponding optical depth also shows highest values close to the mountain crest with decreasing values downstream. The high values of the optical depth close to the mountain crest are caused by the high ICNB as well as the high IWP in this region. The high number concentration of ice crystals in the formation region leads to small $D_{\mathrm{e}}$ and thus increases the optical depth (see Eq. 1). Additionally, the mean IWP, ICNB and optical depth are shown in Fig. A1. The mean is calculated over all values where the optical depth exceeds 0.03 , which distinguishes visible from subvisible cirrus clouds. The mean ICNB amounts to $2.64 \times 10^{8} \mathrm{~m}^{-2}$, the mean IWP to $4.22 \mathrm{~g} \mathrm{~m}^{-2}$ and the mean optical depth to 0.57. In the left column of Fig. 5, the SCF, LCF and NCF are shown for the time period from 06:00 to 12:00 LT. As long as no cloud is present, the cloud forcing equals zero as there is no difference to the clear-sky radiation. When the cloud starts to form after $\sim 3 \mathrm{~h}$ the shortwave 
cloud forcing (SCF) becomes negative, because, due to the presence of the cloud, more radiation is scattered back compared to the clear-sky case. The amount of scattered radiation depends on the optical depth of the cloud (compare to Fig. 4c). The higher the optical depth, the more incoming radiation scattered and the more negative the SCF. The second small updraft region in the wave where the crystals start to grow can again be seen in the optical depth as well as the SCF. The SCF decreases to values below $-150 \mathrm{~W} \mathrm{~m}^{-2}$ close to the mountain crest, where the optical depth is highest and increases with decreasing optical depth. The mean SCF averaged over all values where $\tau>0.03$ amounts to $-48.7 \mathrm{~W} \mathrm{~m}^{-2}$. The longwave cloud forcing (LCF) shows a similar pattern. The highest values of more than $110 \mathrm{~W} \mathrm{~m}^{-2}$ are reached close to the mountain crest, where the IWP has its maximum values and the pattern is again determined by the optical depth of the cloud. The mean LCF is given by $45.6 \mathrm{~W} \mathrm{~m}^{-2}$ and is somewhat lower than the absolute value of the SCF. The mean NCF is therefore slightly negative $\left(-3.1 \mathrm{~W} \mathrm{~m}^{-2}\right)$ such that the cloud is slightly cooling compared to the clear-sky case. However, it can be seen that parts of the cloud are cooling whereas other parts have a warming effect. In the part of the cloud with the highest optical depth close to the mountain crest, the SCF strongly dominates the LCF, leading to a cooling, whereas in regions where the optical depth is lower $(\tau<\sim 0.7)$, the LCF slightly dominates and the cloud is in a warming regime. In the following section it is shown how these microphysical and optical properties change if $10 \mathrm{INL}^{-1}$ that freeze at $\mathrm{RHi}_{\text {het }}=130 \%$ are added and heterogeneous freezing is active as well.

\subsubsection{Simulation $10 \mathrm{IN}$}

In the simulation presented here, $10 \mathrm{INL}^{-1}$ that freeze at $\mathrm{RHi}_{\text {het }}=130 \%$ are added to the background aerosol concentration such that the influence of heterogeneous freezing on the microphysical and optical properties can be investigated. In the right column of Fig. 4 the ICNB, IWP and optical depth are shown as an evolution in time. As the heterogeneous freezing starts at lower RHi than the homogeneous freezing threshold (namely at the prescribed $130 \%$ ), the formation of the cloud already starts after $\sim 60 \mathrm{~min}$, which is $\sim 120$ min earlier than in HOM. Additionally, the slight lifting of the streamlines at the windward side of the mountain is enough to reach supersaturation as needed for heterogeneous freezing. The cloud is therefore no longer constrained to the downstream side of the mountain but extends $\sim 40 \mathrm{~km}$ upstream. The existence of $10 \mathrm{IN} \mathrm{L}^{-1}$ is enough to deplete part of the supersaturation such that the onset of homogeneous freezing is delayed by about $20 \mathrm{~min}$ compared to HOM. The green lines in Fig. 4d and e show the ICNB and IWP, respectively, formed by homogeneous freezing. It can be seen that the onset of homogeneous freezing is slightly shifted in time and that the strength of the homogeneous nucleation event is reduced compared to HOM. This behaviour corresponds very well to the findings of Spichtinger and Cziczo (2010). The heterogeneously frozen ice crystals form in an area with high supersaturation. As there are only few ice crystals, they rapidly grow and start to sediment. Therefore a vertical gradient in the ICNC builds up with more ice crystals at the bottom and fewer ice crystals at the top of the ISSR. The heterogeneously frozen ice crystals further deplete part of the supersaturation, mainly at the bottom of the cloud. Therefore the threshold for homogeneous freezing is only reached in a shallow area close to the top of the ISSR and not throughout the whole depth like in HOM. This effect can be seen in Fig. 6, where the homogeneously frozen crystals, the heterogeneously frozen crystals, and the points where RHi exceeds the critical supersaturation needed for homogeneous freezing are displayed for both simulations (HOM, 10 IN). A strong vertical gradient in the heterogeneously frozen crystals builds up (black lines in Fig. 6, right) with ICNC of $\sim 10 \mathrm{~L}^{-1}$ at the bottom and $\sim 1 \mathrm{~L}^{-1}$ at the top of the cloud. Furthermore, it can be seen that the peak values of RHi above the homogeneous nucleation threshold are smaller in 10 IN than in HOM, at least for the beginning of the homogeneous freezing event (see crosses in Fig. 6). The threshold is only exceeded in a much smaller area close to the cloud top compared to HOM. Due to these two effects, the vertically integrated number of homogeneously frozen ice crystals is smaller in the 10 IN simulation than in HOM. Spichtinger and Gierens (2009c) observed the effect of a reduced nucleation area and smaller peak values in their simulations of a cirrus cloud which forms under much lower vertical velocities of the order of some $\mathrm{cm} \mathrm{s}^{-1}$, i.e. under synoptic updraft conditions. However, the results shown here suggest that this effect is even present in a highly dynamically dominated regime with high updraft velocities and that even a few IN are enough to change the clouds microphysical properties. After $\sim 240 \mathrm{~min}$ the homogeneous nucleation takes place over the whole depth of the ISSR, with a delay of $\sim 60 \mathrm{~min}$ compared to HOM. At that time, and for the rest of the simulation, the remaining heterogeneously frozen ice crystals also cannot inhibit the onset of homogeneous freezing because the high updraft speeds in the mountain wave are shown to be a strong source of cooling and thus the formation of ice supersaturation. In summary, the presence of heterogeneous IN strongly modifies the microphysical properties of the cloud. The formation of homogeneously frozen ice crystals is suppressed and the few heterogeneously frozen ice crystals are relatively large and start to sediment. This leads to the observed reduction in ICNB as seen in the right column of Fig. 4. Due to the reduced ICNB the IWP is also decreased, because large crystals sediment and sublimate, exhibiting a sink for the IWP. Fewer ice crystals and less IWP influence the optical depth, both in the same direction. Fewer but larger ice crystals lead to a decreased $\tau$, as does a decreased IWP (see Eq. 1). The optical depth is therefore strongly reduced compared to HOM, from 0.57 to 0.22 , in the mean over the cloud. In the right column of Fig. 5 the 

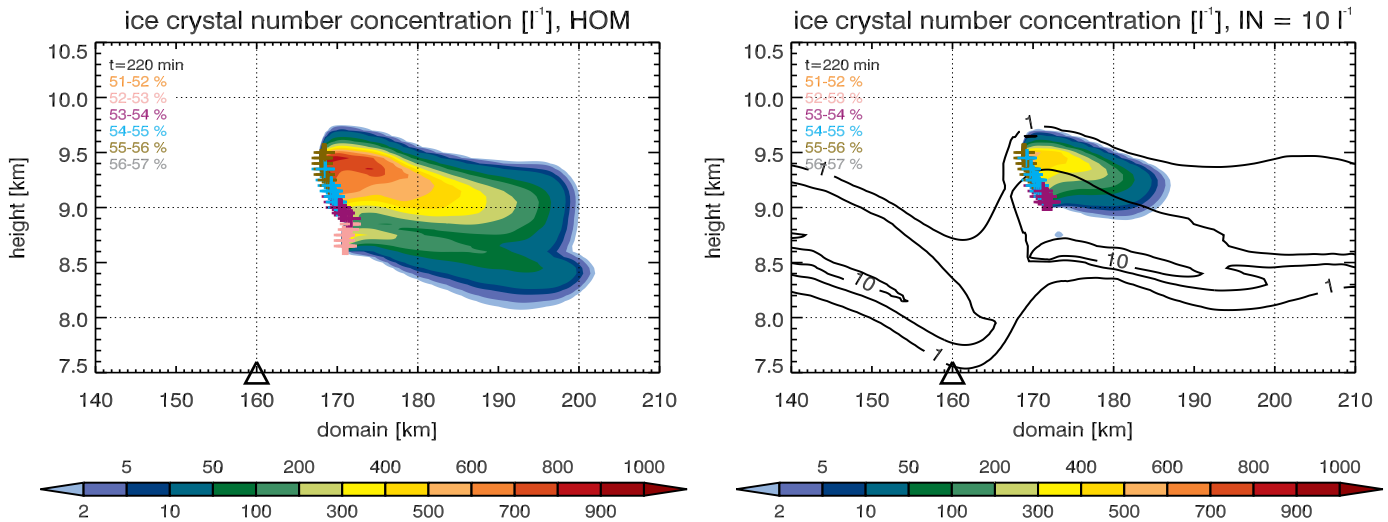

Figure 6. Ice crystal number concentration for simulation HOM (left) and $10 \mathrm{IN}$ (right) for $t=220$ min. Colours denote the homogeneously frozen ice crystals and black lines the heterogeneously frozen ice crystals, with lines for $1,5,10$ and $20 \mathrm{~L}^{-1}$. The crosses show where RHi exceeds the critical supersaturation for homogeneous freezing at this point in time, and the colours show the amount of supersaturation.

corresponding SCF, LCF and NCF are shown. The decreased optical depth leads to a reduced SCF as less radiation is scattered back into space compared to HOM. The peak values of $\sim-150 \mathrm{~W} \mathrm{~m}^{-2}$ are only reached in a much smaller area than in HOM. The part of the cloud where only heterogeneous freezing is active also influences the radiation as the optical depth is still high enough (above the threshold for subvisible cirrus, i.e. $\tau>0.03$ ). Thus, a low negative SCF with values up to $-10 \mathrm{~W} \mathrm{~m}^{-2}$ can be seen in these regions. The mean SCF is $-19.8 \mathrm{~W} \mathrm{~m}^{-2}$, which is much less than the SCF in HOM $\left(-48.7 \mathrm{~W} \mathrm{~m}^{-2}\right)$. The LCF exhibits a similar pattern since IWP is also strongly reduced. The slight upward motion of the streamlines in front of the mountain leads to a non-zero LCF up to $\sim 40 \mathrm{~km}$ upstream of the mountain crest. Due to the rapid growth of ice crystals, which produces IWP values up to $4 \mathrm{~g} \mathrm{~m}^{-2}$, the $\mathrm{LCF}$ reaches values of $\sim 20 \mathrm{~W} \mathrm{~m}^{-2}$ in front of the mountain. On the other side of the mountain, the LCF is reduced in amount because of the strengthened sedimentation of the larger ice crystals compared to HOM. The mean LCF amounts to $19.6 \mathrm{~W} \mathrm{~m}^{-2}$, which corresponds to less than half of the LCF in HOM $\left(45.6 \mathrm{~W} \mathrm{~m}^{-2}\right)$. The difference between SCF and LCF decreases, leading to a less negative NCF compared to HOM. In the updraft region of the wave where the clouds form, the optical depth is still high enough for the SCF to dominate and the cloud is in a cooling regime. However, the cooling region is smaller compared to HOM and the peak values are less pronounced. In the other parts of the clouds, the NCF becomes positive, leading to a warming. However, the absolute values of the warming are less distinct, such that the averaged NCF is given by $-0.2 \mathrm{~W} \mathrm{~m}^{-2}$, i.e. close to zero. Based on the results shown here, we can conclude that a few IN are enough to strongly change the clouds microphysical and radiative properties, even in this dynamically dominated regime. Although the high updraft speeds in the wave lead to strong supersaturations with respect to ice, a few heterogeneous IN are enough to suppress or delay the onset of homogeneous freezing, at least in parts of the cloud, which in turn modifies the radiative properties of the cloud.

\subsection{Summary of all simulations}

In the previous section, two simulations were explained in detail. In order to give an overview of all simulations, in the following only the mean values of the ice crystal number burden, ice water path, optical depth, LCF, SCF and NCF averaged over all values where the optical depth exceeds 0.03 are shown for all 38 radiation calculations.

\subsubsection{Microphysical properties}

Figure 7 shows the summarised results for the microphysical properties ICNB, IWP and optical depth.

The ICNB exhibits a strong dependence on the temperature inside the ISSR (see blue, black and red symbols in Fig. 7 for each simulation). Cold temperatures lead to a decreased crystal growth rate, and as a result the newly formed crystals can only deplete part of the existing supersaturation and new crystals can form. For the warm temperatures the growth of ice crystals is efficient enough to deplete the supersaturation and thus far fewer new crystals form. Adding heterogeneous IN that freeze at an RHi value of $130 \%$ leads to a reduction of the simulated ICNB. As explained in Sect. 3.1.2 the existence of IN that freeze at lower RHi than the homogeneous threshold leads to a suppression or delay of homogeneous freezing and therefore, in total, fewer crystals form. This reduction depends on temperature. For cold temperatures, the relative change is less pronounced than for warm temperatures. The existing IN start to freeze at $\mathrm{RHi}=130 \%$; however, due to the cold temperatures the heterogeneously frozen crystals cannot deplete enough supersaturation since the growth rates are quite low and new crystals can still form homogeneously. At higher temperatures, the relative 

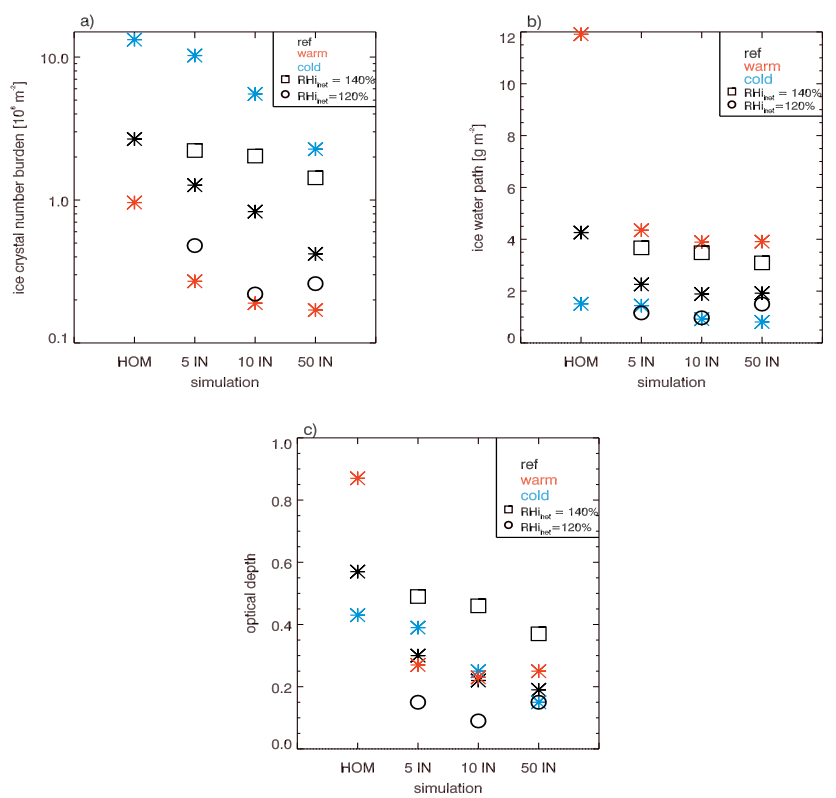

Figure 7. Averaged ice crystal number burden (a), ice water path (b) and optical depth (c) for the simulations HOM, 5 IN, 10 IN and 50 IN. Black, red and blue asterisks show the results for the reference, warm and cold temperature profiles for the simulation where the IN freeze at a critical supersaturation of $130 \%$. Additionally, the results where $\mathrm{RHi}_{\text {het }}$ is increased to $140 \%$ (black squares) or decreased to $120 \%$ (black circles) are shown for the reference temperature profile. For the calculation of the mean, only values where $\tau>0.03$ are considered.

reduction is more pronounced as the heterogeneously formed crystals grow more rapidly and more water vapour is depleted due to efficient diffusion. This leads to a more pronounced suppression of homogeneous freezing. This effect can be seen in Fig. 8, which shows RHi, homogeneously and heterogeneously frozen ice crystals, and the area where homogeneous freezing can occur (i.e. RHi $>$ Scr (critical supersaturation, corresponding to a homogeneous nucleation rate of $J=10^{10} \mathrm{~cm}^{-3} \mathrm{~s}^{-1}$; Kärcher and Lohmann, 2002)) for the simulations HOM and $10 \mathrm{IN}$ for the cold and warm temperature profiles, respectively. It can be seen that the difference in RHi and ICNC between the HOM and 10 IN simulations in the cold temperature case is smaller in $10 \mathrm{IN}$ compared to HOM than in the warm temperature case. In the warm case, $\mathrm{RHi}$ is much lower because it has been depleted by the existing ice crystals. As a result, RHi exceeds Scr only in a very small area (see blue lines) and almost no crystals form homogeneously. In contrast, in the cold case, heterogeneously formed crystals cannot deplete enough RHi to suppress the homogeneous freezing and many crystals can still form homogeneously. For the warm and reference case, the largest reduction occurs between the HOM and 5 IN simulations, whereas for the cold case the difference between $5 \mathrm{IN}$ and $10 \mathrm{IN}$ is larger. The black circles and squares show the results for simulations with the reference temperature profile, but $\mathrm{RHi}_{\text {het }}$ is set to $140 \%$ (squares) and $120 \%$ (circles) in order to test the sensitivity of the results on the efficiency of the heterogeneous IN. If $\mathrm{RHi}_{\text {het }}$ is increased to $140 \%$, the reduction of ICNB is less pronounced as the heterogeneous freezing starts later. Thus, heterogeneously formed ice crystals can grow in a shorter time until homogeneous nucleation initiates. This leads to less efficient water vapour depletion, and the proceeding homogeneous nucleation is suppressed only weakly. Suppression is more pronounced for the $120 \%$ case, as heterogeneous freezing starts there immediately, which leads to a strong suppression of homogeneous freezing due to efficient depletion of water vapour. The simulated IWP depends strongly on the assumed temperature profile. As we initialised the model with an ice-supersaturated region of $\mathrm{RHi}=120 \%$, the shift of the temperature profile leads to a varying specific humidity inside the ISSR, with highest values for the warm case. Therefore, the simulated IWP strongly depends on the assumed temperature profile with highest values for the warm case where most water vapour is available (see Fig. 7b). The IWP decreases strongly with decreasing temperatures. The addition of heterogenous IN also leads to a strong reduction in the IWP as fewer crystals form. The available water vapour is depleted on the reduced number of ice crystals, which then become large enough to sediment. The sedimenting ice crystals start to sublimate, exhibiting a sink for the IWP. The change in IWP is strongest between the HOM and 5 IN simulations, whereas less change is seen from $5 \mathrm{IN}$ to $50 \mathrm{IN}$. The change of $\mathrm{RHi}_{\text {het }}$ to $140 \%$ (120\%) leads to a less (more) pronounced difference between HOM and $5 \mathrm{IN}$ because more (less) water vapour is depleted due to the increase (decrease) in ICNB. The optical depth shown in Fig. 7c depends on both the ICNB (or the effective ice crystal size) and the IWP. For the simulation HOM it can be seen that the high IWP in the warm case dominates the much lower ICNB, leading to the highest optical depth for the warm case and the lowest optical depth for the cold case. As the addition of heterogeneous IN leads to a strong decrease in IWP and a decrease in ICNB, the resulting optical depth is characterised by decreasing values with increasing IN concentration. Again, the difference is largest from HOM to $5 \mathrm{IN}$ and the further decrease in optical depth with increasing IN is much less distinct. Further, the shift of $\mathrm{RHi}_{\text {het }}$ to $140 \%(120 \%)$ leads to a less (more) pronounced decrease according to the behaviour of the IWP and ICNB (see black circles and squares in Fig. 7a, b).

\subsubsection{Cloud forcing}

In Fig. 9 the SCF, LCF and NCF for all simulations are summarised. The different symbols show the results for the simulations from 06:00 to 12:00 LT (asterisks) and from 12:00 to 18:00 LT (diamonds). In addition, the results for the reference temperature profile and $\mathrm{RHi}_{\text {het }}=140 \%(120 \%)$ are shown as black squares (circles) for the time period 06:00 to 12:00 LT only. 

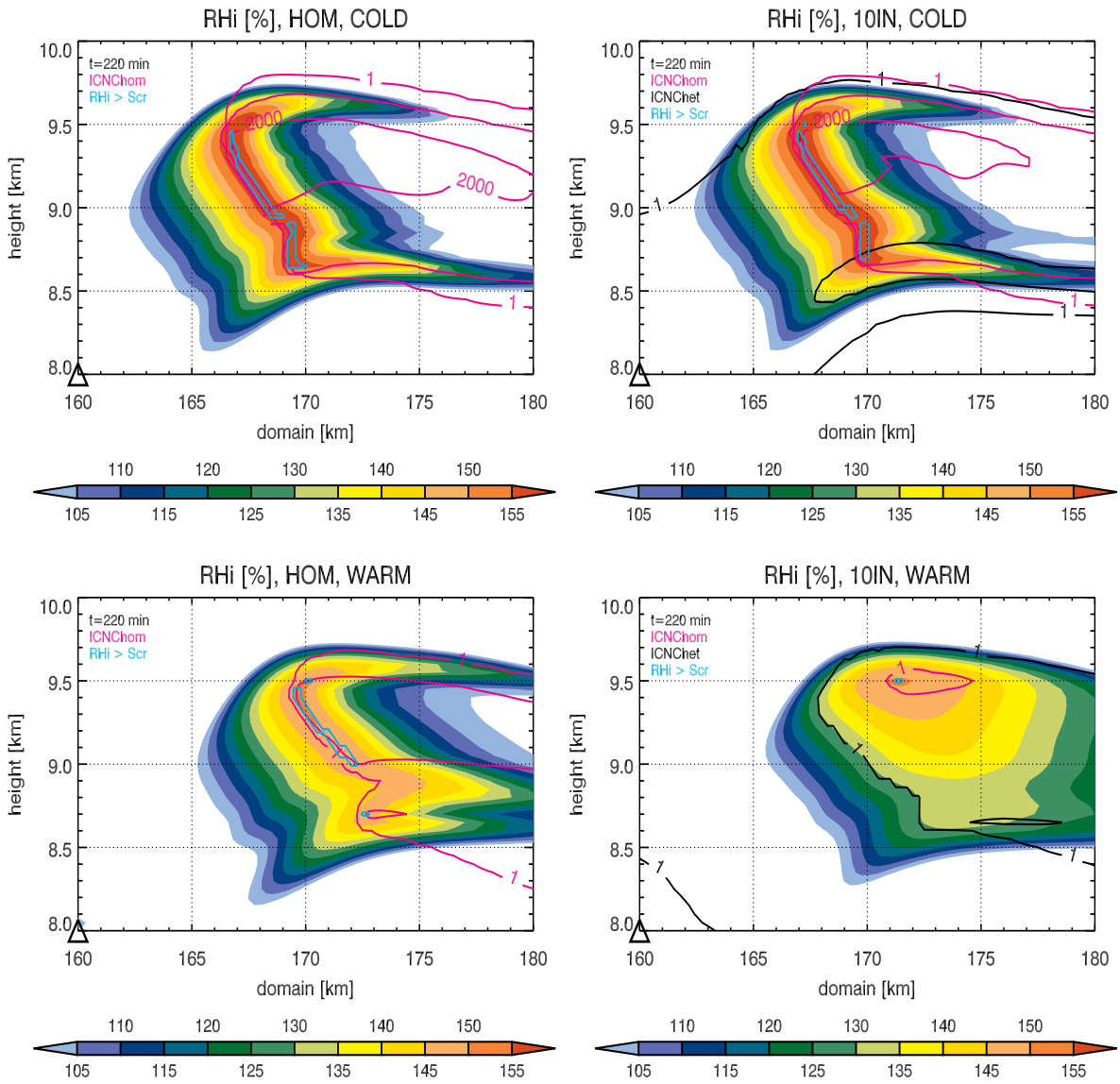

Figure 8. Relative humidity with respect to ice (RHi) for the simulations HOM (left column) and 10 IN (right column), for the cold (upper row) and warm (lower row) temperature profiles for $t=220 \mathrm{~min}$. Colours denote RHi in percent; black lines the heterogeneously frozen ice crystals with lines for 1 and $10 \mathrm{~L}^{-1}$; pink lines the homogeneously frozen crystals with lines for 1,100 and $2000 \mathrm{~L}^{-1}$; and the blue dashed area shows where RHi exceeds the critical supersaturation for homogeneous freezing $S_{\mathrm{cr}}=2.583-\frac{T}{207.83}(\mathrm{see}$ Kärcher and Lohmann (2002)).

\section{Shortwave cloud forcing, SCF}

Figure 9a shows the SCF. In all simulations, the most negative values appear for the simulations with the highest optical depth. Thus, for HOM, the warm case reveals the most negative SCF. With decreasing optical depth, SCF decreases in all simulations, as does the spread between the reference, cold and warm simulations. The most pronounced difference in SCF occurs between HOM and 5 IN for the warm and reference cases, whereas the change in SCF from $5 \mathrm{IN}$ to $50 \mathrm{IN}$ is much smaller. For the cold case, the largest difference occurs from $5 \mathrm{IN}$ to $10 \mathrm{IN}$. Additionally it can be seen that the simulations from 06:00 to 12:00 LT show more negative values than the simulations from 12:00 to $18: 00 \mathrm{LT}$. This is caused by the increasing zenith angle as time progresses. The simulations start at 12:00 LT; thus the clouds start to form between $\sim$ 13:00 and 15:00 LT depending on the IN concentration. At that time the zenith angle is still small enough to produce considerable scattering of radiation back into space, whereas later the SCF starts to decrease because of the in- crease in zenith angle, although the highest optical depth is reached around 18:00LT (not shown). The mean over the whole cloud where $\tau>0.03$ is therefore smaller than for the simulation from 06:00 to 12:00 LT where the clouds are formed at $\sim$ 07:00 to 09:00 LT depending on the IN concentration. For better illustration, the diurnal cycle of the SCF for a single-column simulation with a cirrus cloud with a mean ice water content of $2 \mathrm{mg} \mathrm{m}^{-3}$ between 8 and $9 \mathrm{~km}$ altitude, representative of mid-latitude cirrus (Schiller et al., 2008), as well as an idealised temperature profile, is shown in Fig. 10. During the night, the SCF is zero and starts to become negative after sunrise with decreasing solar zenith angle. The radiation calculations are done for different days of the year, namely 21 March, 21 June and 21 December. For spring and summer, it can be seen that the minimum in SCF is not reached at noon at the smallest zenith angle but that it is smaller in the morning and afternoon. This effect was also found by Meerkötter et al. (1999) (see their Fig. 4a). The exact values of the SCF depend of course on the temperature and humidity profiles and the assumed IWC. 

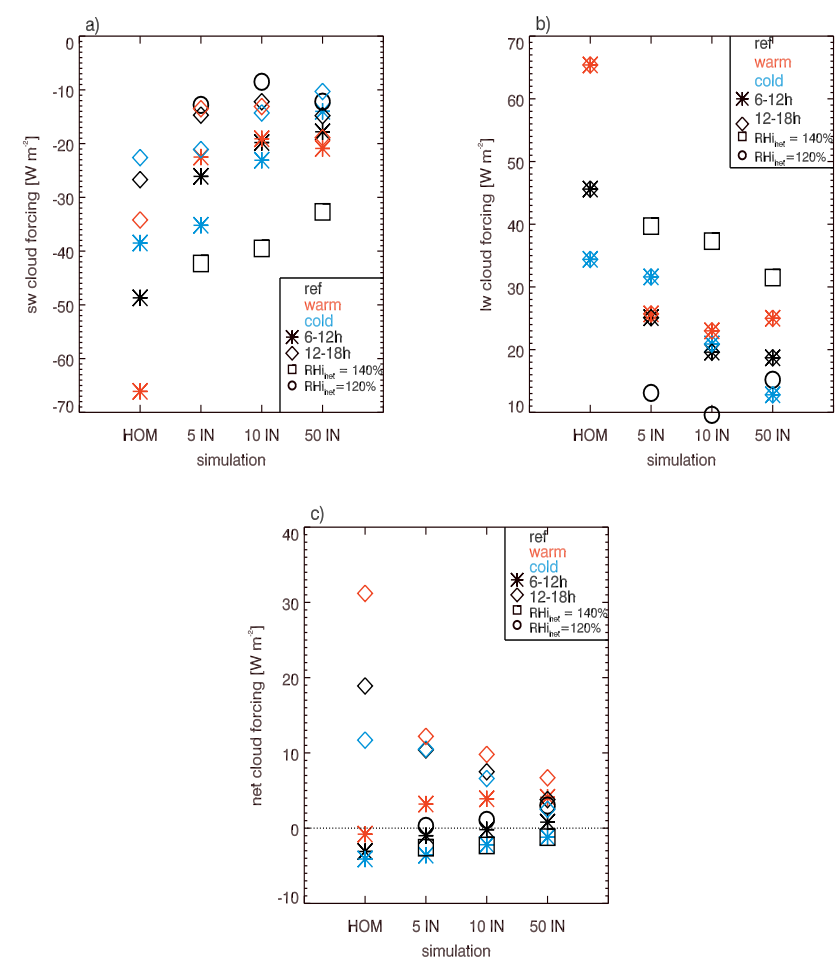

Figure 9. Averaged shortwave cloud forcing (a), longwave cloud forcing (b) and net cloud forcing (c) for the simulations HOM, 5 IN, $10 \mathrm{IN}$ and $50 \mathrm{IN}$. Black, red and blue asterisks (diamonds) show the results for the reference, warm and cold temperature profiles for 06:00-12:00 LT (asterisks) and 12:00-18:00 LT (diamonds) for the simulation where the IN freeze at $\mathrm{Rhi}_{\text {het }}=130 \%$. Additionally, the results where $\mathrm{RHi}_{\text {het }}$ is increased to $140 \%$ (black square) or decreased to $120 \%$ (black circles) are shown for the reference temperature profile and the time period from 06:00 to 12:00 LT. For the calculation of the mean, only values where $\tau>0.03$ are considered.

However, with Fig. 10 we want to highlight the general behaviour of the SCF during a diurnal cycle. The simulations with $\mathrm{RHi}_{\text {het }}=140 \% / 120 \%$ reveal the same behaviour as the optical depth, thus more negative SCF for $\mathrm{RHi}_{\text {het }}=140 \%$ where the optical depth is higher than for the reference case and less negative SCF for $\mathrm{RHi}_{\text {het }}=120 \%$ where the optical depth is strongly decreased by the very efficient heterogeneous IN, suppressing homogeneous nucleation.

\section{Longwave and net cloud forcing, LCF+NCF}

The LCF shown in Fig. 9b is of course independent of the simulated time of day. Like the SCF it strongly depends on the optical depth. The strongest LCF can be seen for the simulation HOM and the warm case, whereas it decreases with increasing IN and decreasing temperature in the ISSR. This would also mean that during night, when there is no contribution from the shortwave spectrum, the clouds become less warming with increasing IN concentration. The result-

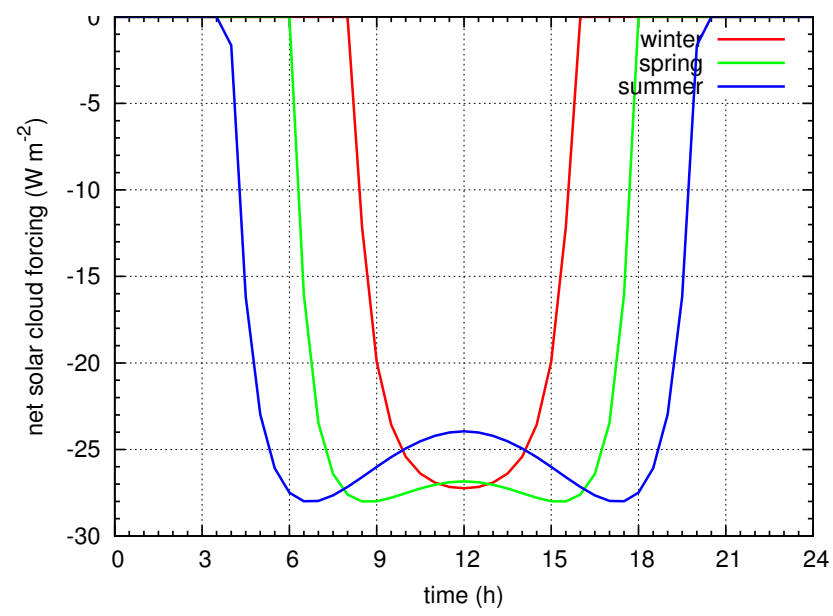

Figure 10. Diurnal cycle of SCF for zenith angles calculated for 21 March (spring, green), 21 June (summer, blue) and 21 December (winter, red) for $50^{\circ} \mathrm{N}$.

ing NCF is shown in Fig. 9c. Here, a clear distinction can be seen between the NCF for the different simulated times of day. The simulation from 06:00 to 12:00 LT reveals negative and positive NCF values depending on the temperature and number of IN in the simulation. The most negative NCF can be seen for the simulation HOM and the cold temperature case. In this case, the SCF caused by many small crystals dominates the LCF, leading to the negative NCF of $-4.2 \mathrm{~W} \mathrm{~m}^{-2}$. The negative NCF is reduced with increasing temperatures and increasing IN concentration. For the warm case, a transition from the cooling to the warming regime already begins to take place from HOM to $5 \mathrm{IN}$, whereas the cold case is always cooling and the reference case becomes slightly positive (warming) only for the 50 IN simulation. The simulations with $\mathrm{RHi}_{\text {het }}=140 \%, 120 \%$ show a similar behaviour to the reference simulation, whereas the difference to the simulation HOM is decreased (increased) for $\mathrm{RHi}_{\text {het }}=140 \%(120 \%)$ where the heterogeneous freezing is less (more) efficient. The simulations from 12:00 to 18:00 lead to a positive NCF. This is caused by the reduced SCF due to the increased zenith angle at the end of the evolution of the cloud where the optical depth is highest. As the LCF is the same for all times of day, the reduction of SCF leads to a positive NCF. With decreasing temperature and increasing IN concentration, the NCF is strongly reduced, whereas the largest change for the reference and warm temperature profile occurs from HOM to $5 \mathrm{IN}$, but only smaller changes occur for the cold case. In general we might conclude that the same cloud can lead to a warming or cooling depending on the times of day when it starts to form. For example, in HOM, the same microphysical properties lead to an NCF of $-4.2 \mathrm{~W} \mathrm{~m}^{-2}$ for the cold case between $06: 00$ and 12:00 LT, whereas it leads to a positive NCF of $11.7 \mathrm{~W} \mathrm{~m}^{-2}$ for the 12:00 to 18:00 simulation. Additionally, the cloud can shift from a cooling into a warming regime depending on the 

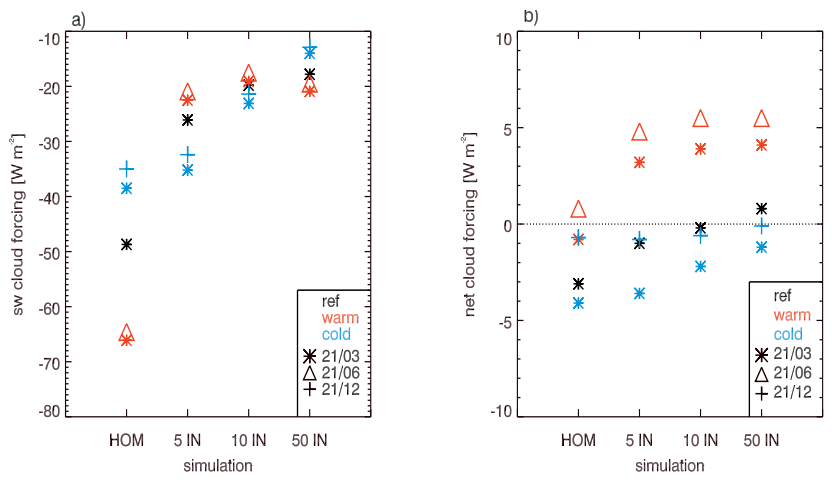

Figure 11. Averaged shortwave cloud forcing (a) and net cloud forcing (b) for the simulations HOM, 5 IN, 10 IN and 50 IN. Black, red and blue asterisks show the results for the reference, warm and cold temperature profiles for 06:00-12:00 LT for the simulation where the IN freeze at $\mathrm{RHi}_{\text {het }}=130 \%$ and the solar zenith angle is representative of $21 \mathrm{March}$. Additionally, the results where the solar zenith angel is representative of 21 June (21 December) are shown for the warm (cold) profile for 06:00-12:00 LT (red triangles, blue crosses) and $\mathrm{RHi}_{\text {het }}=130 \%$. For the calculation of the mean, only values where $\tau>0.03$ are considered.

number of heterogeneous IN. Based on our simulations, it seems that the presence of even a few IN of the order of $5 \mathrm{~L}^{-1}$ is enough to strongly modify the clouds microphysical and radiative properties. A further increase in the IN concentration from 5 to $50 \mathrm{~L}^{-1}$ reveals a much smaller change. It needs to be emphasised here that these results are only valid for the case in which there is no feedback from the radiation to the microphysics. However, since we are investigating strongly stratified simulations, the radiation feedback will probably not impact the underlying dynamics. Thus, the quality of the results should not change.

\section{Seasonal dependence}

The warm (cold) temperature profiles correspond to a surface temperature of $298 \mathrm{~K}(270 \mathrm{~K})$, representative of the northern hemispheric mid-latitude summer (winter). We therefore performed additional simulations where the solar zenith angle is representative of 21 June (21 December) according to the surface temperatures. The simulations are performed for 06:00-12:00 LT and with $\mathrm{RHi}_{\text {het }}=130 \%$. The results can be seen in Fig. 11. For a better comparison, the results for the simulations from 06:00 to $12: 00 \mathrm{LT}$, $\mathrm{RHi}_{\text {het }}=130 \%$ and a solar zenith angle for 21 March are also included. In general, the same behaviour as for the simulations with the zenith angle representative of 21 March can be seen, with a decreasing SCF with increasing IN concentration. The simulations with the warm profile for 21 June (red triangles) show a reduced SCF compared to the simulation with the same temperature profile but a zenith angle calculated for 21 March (red asterisks). This effect is caused by the fact that the SCF reaches the most negative values in the morning hours instead of noon. The cloud develops between 06:00 and 12:00 LT and reaches the highest optical depth at the end of the simulation time around noon. As, due to the geometry of the ice crystals (columns), the SCF is less increased around noon for the March simulation than for the June simulation, the mean SCF over the $6 \mathrm{~h}$ simulation is slightly less negative for June than for March (see also Fig. 10, blue and green lines). The resulting NCF is therefore more positive for the June simulation than that of March. The cold simulation for 21 December shows slightly lower SCF values because of the lower solar altitude in December compared to March, although the overall behaviour stays the same. This reduced SCF leads to a lower NCF for the December simulation, and the clouds are less cooling than for the March simulation.

\section{Summary and conclusions}

Idealised simulations with the large eddy simulation model EULAG (Prusa et al., 2008), including state-of-the-art bulk ice microphysics and a radiative transfer code (Fu and Liou, 1993), have been performed in order to investigate the influence of heterogeneous freezing on the microphysical and radiative properties of orographic cirrus clouds. We performed 2-D simulations of orographic cirrus clouds in a vertically propagating wave with homogeneous freezing only and simulations with heterogeneous and homogeneous freezing, for which the number of heterogeneous IN was set to 5,10 and $50 \mathrm{IN} \mathrm{L}^{-1}$. The results of the simulated ice water content and ice crystal number concentration are used as an input for the radiation transfer code, and the optical depth as well as the TOA radiative fluxes for the thermal and solar spectrum are calculated. The LES simulations were run for $6 \mathrm{~h}$. During that time a stable flow regime established and an orographic cirrus cloud started to form. The output frequency for the microphysical properties calculated with the EULAG model is $10 \mathrm{~min}$, and thus the input fields needed by the radiation code are also available every $10 \mathrm{~min}$. We therefore obtained a time series of microphysical and radiative properties with a time resolution of $10 \mathrm{~min}$ over $6 \mathrm{~h}$. Additionally, we performed simulations where the temperature inside the ISSR was increased/decreased by $10 \mathrm{~K}$ in order to investigate the dependence of the results on the temperature in the cirrus layer. The radiation calculation was done for two different time periods (06:00 to 12:00 and 12:00 to 18:00 LT), while the solar zenith angle, which is representative of $50^{\circ} \mathrm{N}$ and the 21 March, was updated every $10 \mathrm{~min}$, together with the microphysical input, in order to test the dependence of the results on the time of day. Moreover, the critical relative humidity $\mathrm{RHi}_{\text {het }}$ at which the heterogeneous freezing starts is shifted to 120 and $140 \%$ for the simulation from 06:00 to 12:00 LT and the reference temperature profile. Additional simulations for HOM, 5 IN, 10 IN and 50 IN were performed where a zenith angle representative of 21 June (21 December) is used for 
the warm (cold) temperature profile as the surface temperature corresponds to summer (winter) conditions for the warm (cold) profiles. In total we therefore obtain 38 radiative calculation scenarios (see Fig. 3). The main findings of these 38 scenarios can be summarised as follows:

\section{Microphysical changes}

- The ICNB, IWP and optical depth strongly decrease even in the presence of only a few IN $\left(5 \mathrm{~L}^{-1}\right)$ in a highly dynamical regime.

- The largest changes occur from HOM to 5 IN in most of the simulations, whereas the changes from 5 IN to $50 \mathrm{IN}$ are much smaller.

- The decrease is strongest for warmer temperatures because of the faster growth of the ice crystals and the strong suppression of the homogeneous freezing.

- The decrease strongly depends on the efficiency of the IN.

\section{Radiative changes}

- The absolute value of the NCF strongly decreases with increasing IN.

- The same cirrus cloud can result in a net warming or cooling depending on the time of day when it forms.

- The presence of even a few IN can shift the cloud from a cooling to a warming regime.

- During the night (only LCF), the clouds become less warming with increasing IN concentration.

\section{Seasonal changes}

- Winter cirrus are less cooling compared to the same cirrus forming in spring (reference case) due to the decreased SCF.

- Summer cirrus are slightly more warming compared to the same cirrus forming in spring (reference case) due to the decreased SCF in summer around noon.
These results emphasise the complexity of processes that have to be taken into account when the radiative effect of orographic cirrus (or cirrus in general) is calculated. Joos et al. (2009) demonstrated the dependence of the microphysical properties on the underlying dynamics as well as thermodynamic conditions under which the cloud forms. Together with the results presented here, we want to illustrate that, in order to predict whether a cloud is warming or cooling, it is necessary to predict the correct dynamical forcing of the cloud, the correct thermodynamical conditions, the availability and efficiency of heterogeneous IN, and the time of day. A change in the anthropogenically produced IN concentration in future might have an influence on the radiative properties of cirrus clouds if more IN were transported to the upper troposphere. If wrong conditions for the cloud formation are taken into account, the cloud forcing can change from a warming to a cooling regime or strongly change the absolute value. This might be the case for general circulation models where the small-scale vertical velocity fluctuations are not resolved by the grid and the exact number concentration and efficiency of IN is not known exactly. Our results suggest that a small error in the predicted IN concentration can lead to a wrong simulation of the cirrus cloud forcing. The aim of this study was to highlight the influence of heterogeneous freezing, temperature and time of day on the microphysical and radiative properties in a highly idealised framework. This setup allows us to investigate the physical mechanisms that lead to the huge scattering in cirrus cloud radiative forcing; however, no quantitative conclusions are possible. A weakness of this study is that the radiation has no feedback on the cloud evolution and life cycle. However, this study provides insight into the complexity of predicting radiative properties of (orographic) cirrus clouds and might be used as motivation for further investigation of the manifold multiscale interactions between dynamics, thermodynamics and cirrus clouds for predicting, for instance, their impact on Earth's radiation budget. 

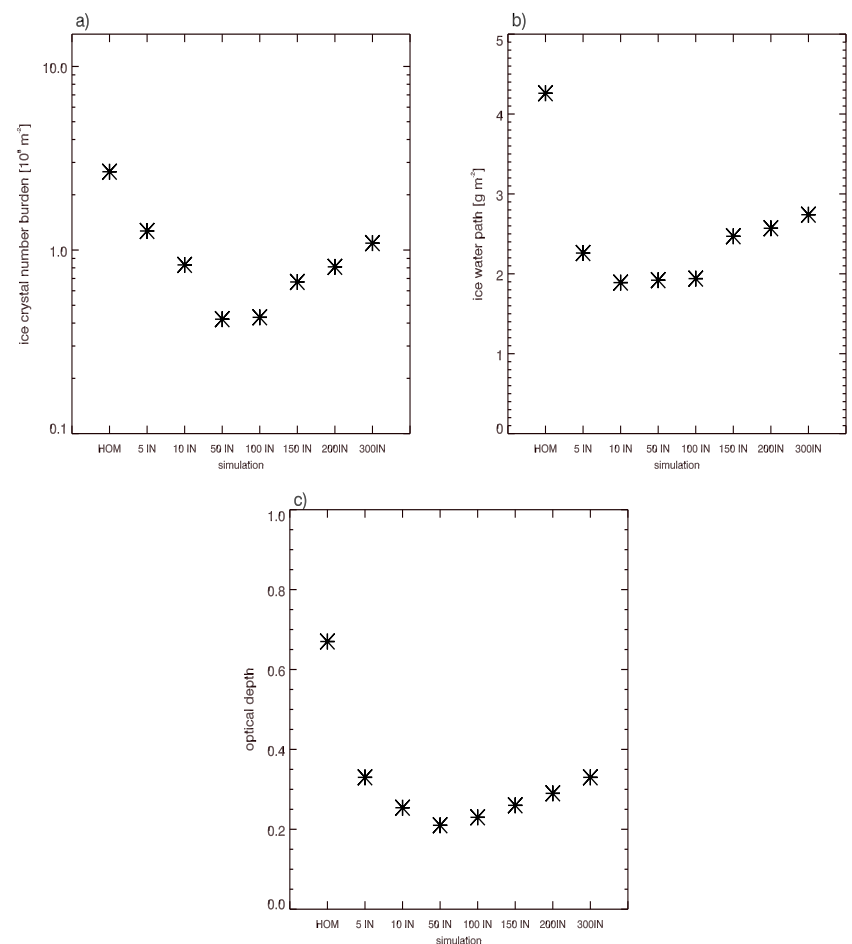

Figure A1. Averaged ice crystal number burden (a), ice water path (b) and optical depth (c) for the simulations HOM, 5 IN, 10 IN, 50 IN, 100 IN, 150 IN, 200 IN and 300 IN for the reference temperature profile for the simulation where the IN freeze at $\mathrm{RHi}_{\text {het }}=130 \%$. For the calculation of the mean, only values where $\tau>0.03$ are considered.

\section{Appendix A: additional simulations with unrealistic high IN concentration}

We performed additional simulations with 100, 150, 200 and $300 \mathrm{IN} \mathrm{L}^{-1}$ for the reference temperature profile and 06:0012:00 LT. Although these high IN concentrations are unrealistic (DeMott et al., 2003), we investigate whether the results for the simulations with very high IN concentration start to approach the simulation HOM and whether there is a turning point in the behaviour. The mean ICNB, IWP and optical depth are shown in Fig. A1. If the IN concentration becomes larger than $50 \mathrm{~L}^{-1}$, the ICNB, IWP and optical depth start to increase again. In the simulations with more than $50 \mathrm{IN} \mathrm{L}^{-1}$, the homogeneous freezing is strongly weakened or even completely suppressed for simulations 200 IN and 300 IN. However, the high IN concentration in these simulations leads to an increase in the heterogeneously frozen ice crystals (ICNB), and thus IWP and optical depth increase. Additionally, one main difference compared to HOM is the fact that a cloud also forms at the windward side of the mountain. Thus, ICNC at the windward side of the mountain is increased compared to HOM, whereas the ICNC above the mountaintop is strongly decreased because homogeneous freezing is strongly weakened/suppressed. Thus, the mono-
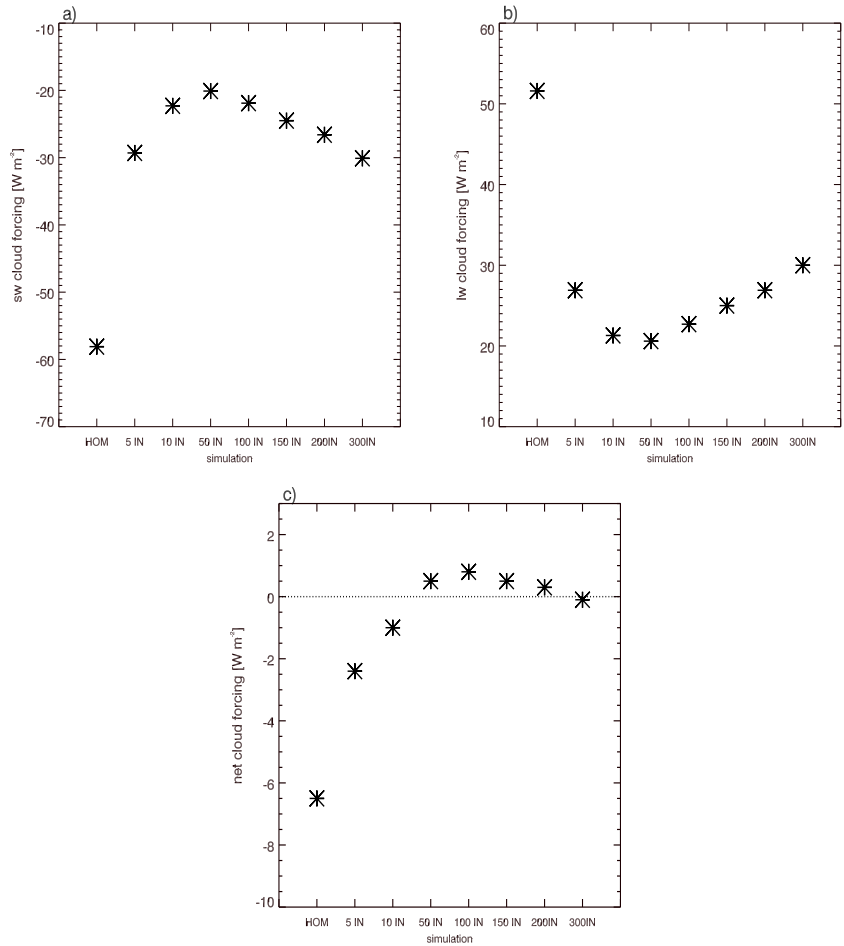

Figure A2. Averaged shortwave cloud forcing (a), longwave cloud forcing (b) and net cloud forcing (c) for the simulations HOM, $5 \mathrm{IN}$, $10 \mathrm{IN}, 50 \mathrm{IN}, 100 \mathrm{IN}, 150 \mathrm{IN}, 200 \mathrm{IN}$ and $300 \mathrm{IN}$ for the reference temperature profiles of 06:00-12:00 LT for the simulation where the IN freeze at $\mathrm{RHi}_{\text {het }}=130 \%$. For the calculation of the mean, only values where $\tau>0.03$ are considered.

tonicity breaks down if the IN exceed a value between 50 and $100 \mathrm{~L}^{-1}$. The SCF, LCF and NCF show the same behaviour (see Fig. A2). In the simulations with more than $50 \mathrm{INL}^{-1}$, the absolute values of SCF and LCF start to increase again. This behaviour is the same as the one for the optical depth. Again, the increase from $50 \mathrm{IN}$ to $300 \mathrm{IN}$ is less pronounced than the decrease from HOM to 50 IN. For the 300 IN simulation, the same mean optical depth as in $5 \mathrm{IN}$ is simulated. However, the NCF does not show the same value in these two simulations. This is caused by the different distribution of the ice crystals in the windward side and above the mountain. In front of the mountain, the NCF is more negative in 300 IN compared to HOM, whereas it is less negative above the mountaintop. This reflects the distribution of ICNB and IWP in these two simulations. 
Appendix B: calculation of the generalised effective ice crystal size

We assume the following general case of an ice crystal size distribution:

$f(L):=f_{1}(L)+f_{2}(L)+\ldots+f_{n}(L)=\sum_{i=1}^{n} f_{i}(L)$.

Here, the distribution type of $f_{i}(L)$ is not specified. However, we have to assume, for all distributions $f_{i}(L), i=1, \ldots, n$, that the generalised moments

$\mu_{k, i}[L]:=\int_{0}^{\infty} L^{k} f_{i}(L) \mathrm{d} L$

for $k \leq 1$ exist. Similarly, we define the generalised truncated moments as

$\mu_{k, i}\left[L, L_{1}, L_{2}\right]:=\int_{L_{1}}^{L_{2}} L^{k} f_{i}(L) \mathrm{d} L$.

Since the integral is a linear operator, the generalised moments and the generalised truncated moments for the whole distribution $f(L)$ can be described as

$\mu_{k}[L]=\int_{0}^{\infty} L^{k} f(L) \mathrm{d} L=$

$\int_{0}^{\infty} \sum_{i=1}^{n} L^{k} f_{i}(L) \mathrm{d} L=\sum_{i=1}^{n} \mu_{k, i}[L]$

$\mu_{k}\left[L, L_{1}, L_{2}\right]=\int_{L_{1}}^{L_{2}} L^{k} f(L) \mathrm{d} L=\int_{L_{1}}^{L_{2}} \sum_{i=1}^{n}$

$L^{k} f_{i}(L) \mathrm{d} L=\sum_{i=1}^{n} \mu_{k, i}\left[L, L_{1}, L_{2}\right]$.

For ice crystals of columnar shape (as described, for example, in Spichtinger and Gierens, 2009a) we can find a power law relationship between mass $m$ and maximum length $L$ via $m=\alpha(m) \cdot L^{\beta(m)}$ with piecewise constant coefficients as follows:

$\alpha(m)=\left\{\begin{array}{lll}\alpha_{1} & \text { for } \quad m \leq m_{\text {crit }} \\ \alpha_{2} & \text { for } & m>m_{\text {crit }}\end{array}\right.$

$\beta(m)=\left\{\begin{array}{lll}\beta_{1} & \text { for } \quad m \leq m_{\text {crit }} \\ \beta_{2} & \text { for } \quad m>m_{\text {crit }}\end{array}\right.$

The diameter $D$ of the columnar-shaped ice crystals can be described using the aspect ratio $r_{\mathrm{a}}:=L / D$, which can be described as

$r_{\mathrm{a}}:=\left\{\begin{array}{lll}1 & \text { for } & m \leq m_{\text {crit }} \\ \sqrt{\frac{\sqrt{27} \cdot \rho_{\mathrm{b}}}{\frac{3}{\beta_{2}}}} \cdot m^{\frac{3-\beta_{2}}{2 \beta_{2}}} & \text { for } & m>m_{\text {crit }} .\end{array}\right.$
Here, $\rho_{\mathrm{b}}=0.81 \cdot 10^{3} \mathrm{~kg} \mathrm{~m}^{-3}$ denotes the bulk ice mass density. The generalised effective size $D_{\mathrm{e}}$ (e.g. Fu and Liou, 1993) can be defined as follows:

$D_{e}:=\frac{\int_{0}^{\infty} D^{2} L f(L) \mathrm{d} L}{\int_{0}^{\infty} D L f(L) \mathrm{d} L}=\frac{\int_{0}^{\infty} D^{2} L f(L) \mathrm{d} L}{\int_{0}^{\infty} D L f(L) \mathrm{d} L}$.

Thus, for the calculation of the effective size we have to find expressions for the quantities $D^{2} L$ and $D L$ for the different ranges in the mass regime (i.e. $m \leq m_{\text {crit }}, m>m_{\text {crit }}$ ):

- $m \leq m_{\text {crit }}$, i.e. $\alpha=\alpha_{1}, \beta=\beta_{1}=3, r_{\mathrm{a}} \equiv 1$ (droxtals): For this regime we obtain the following expressions:

$$
\begin{aligned}
& D=\frac{L}{r_{\mathrm{a}}}=L=\frac{1}{\alpha^{\frac{1}{\beta}}} m^{\frac{1}{\beta}}, \\
& D^{2}=D L=L^{2}=\frac{1}{\alpha^{\frac{2}{\beta}}} m^{\frac{2}{\beta}} \\
& D^{2} L=L^{3}=\frac{1}{\alpha^{\frac{3}{\beta}}} m^{\frac{3}{\beta}} \stackrel{\beta=3}{=} \frac{1}{\alpha} m .
\end{aligned}
$$

- $m>m_{\text {crit }}$, i.e. $\alpha=\alpha_{2}, \beta=\beta_{2}, r_{\mathrm{a}}>1$ (columns): for this regime we obtain the following expressions:

$$
\begin{gathered}
D=\frac{L}{r_{\mathrm{a}}}=\sqrt{\frac{8 \alpha^{\frac{3}{\beta}}}{\sqrt{27} \rho_{\mathrm{b}}} m^{\frac{\beta-3}{2 \beta}} \frac{1}{\alpha^{\frac{1}{\beta}}} m^{\frac{1}{\beta}}} \\
=\frac{1}{\alpha^{\frac{1}{\beta}}} \sqrt{\frac{8 \alpha^{\frac{3}{\beta}}}{\sqrt{27} \rho_{\mathrm{b}}}} m^{\frac{\beta-1}{2 \beta}}, \\
D L=\frac{1}{\alpha^{\frac{1}{\beta}}} \sqrt{\frac{8 \alpha^{\frac{3}{\beta}}}{\sqrt{27} \rho_{\mathrm{b}}} m^{\frac{\beta-1}{2 \beta}} \frac{1}{\alpha^{\frac{1}{\beta}}} m^{\frac{1}{\beta}}} \\
=\frac{1}{\alpha^{\frac{2}{\beta}}} \sqrt{\frac{8 \alpha^{\frac{3}{\beta}}}{\sqrt{27} \rho_{\mathrm{b}}}} m^{\frac{\beta+1}{2 \beta}}, \\
D^{\frac{1}{\beta}} m^{\frac{\beta-1}{\beta}} \frac{1}{\alpha^{\frac{1}{\beta}}} m^{\frac{1}{\beta}}=\frac{8}{\sqrt{27} \rho_{\mathrm{b}}} m .
\end{gathered}
$$

We can then carry out the integration in the mass space, using the transformation law for probability densities $(f(L) \mathrm{d} L=f(m) \mathrm{d} m)$; this leads to the following integrals: 
Thus, we can combine the terms for numerator and de-

$$
\begin{aligned}
\int_{0}^{\infty} D^{2} L f_{i}(L) \mathrm{d} L & =\int_{0}^{\infty} D^{2}(m) L(m) f_{i}(m) \mathrm{d} m, \\
& =\int_{0}^{m_{\text {crit }}} D^{2}(m) L(m) f_{i}(m) \mathrm{d} m \\
& +\int_{m_{\text {crit }}}^{\infty} D^{2}(m) L(m) f_{i}(m) \mathrm{d} m,
\end{aligned}
$$

$=\int_{0}^{m_{\text {crit }}} \frac{1}{\alpha_{1}} m f_{i}(m) \mathrm{d} m+\int_{m_{\text {crit }}}^{\infty} \frac{8}{\sqrt{27} \rho_{\mathrm{b}}} m f_{i}(m) \mathrm{d} m$,

$=\frac{1}{\alpha_{1}} \mu_{i, 1}\left[m, 0, m_{\text {crit }}\right]+\frac{8}{\sqrt{27} \rho_{\mathrm{b}}} \mu_{i, 1}\left[m, m_{\text {crit }}, \infty\right]$,

$\alpha_{1}=\stackrel{\sqrt{27} \rho_{\mathrm{b}}}{=} \frac{8}{\sqrt{27} \rho_{\mathrm{b}}} \mu_{i, 1}[\mathrm{~m}]$,

$\int_{0}^{\infty} \operatorname{DLf}_{i}(L) \mathrm{d} L=\int_{0}^{\infty} D(m) L(m) f_{i}(m) \mathrm{d} m$,

$=\int_{0}^{m_{\text {crit }}} D(m) L(m) f_{i}(m) \mathrm{d} m$,

$+\int_{m_{\text {crit }}}^{\infty} D(m) L(m) f_{i}(m) \mathrm{d} m$,

$=\int_{0}^{m_{\text {crit }}} \frac{1}{\alpha_{1}^{\frac{2}{\beta_{1}}}} m^{\frac{2}{\beta_{1}}} f(m) \mathrm{d} m$,

$+\int_{m_{\text {crit }}}^{\infty} \frac{1}{\alpha_{2}^{\frac{2}{\beta_{2}}}} \sqrt{\frac{8 \alpha_{2}^{\frac{3}{\beta_{2}}}}{\sqrt{27}} \rho_{\mathrm{b}}} m^{\frac{\beta_{2}+1}{2 \beta_{2}}} f(m) \mathrm{d} m$,

$=\frac{1}{\alpha_{1}^{\frac{2}{\beta_{1}}}} \mu_{i, 2 / \beta_{1}}\left[m, 0, m_{\text {crit }}\right]$,

$$
+\frac{1}{\alpha_{2}^{\frac{2}{\beta_{2}}}} \sqrt{\frac{8 \alpha_{2}^{\frac{3}{\beta_{2}}}}{\sqrt{27} \rho_{\mathrm{b}}}} \mu_{i, \frac{\beta_{2}+1}{2 \beta_{2}}}\left[m, m_{\text {crit }}, \infty\right] .
$$
nominator,

$$
\begin{aligned}
I_{1} & :=\frac{8}{\sqrt{27} \rho_{\mathrm{b}}} \sum_{i=1}^{n} \mu_{i, 1}[\mathrm{~m}], \\
I_{2}:= & \frac{1}{\alpha_{1}^{\frac{2}{\beta_{1}}}} \sum_{i=1}^{n} \mu_{i, 2 / \beta_{1}}\left[m, 0, m_{\text {crit }}\right], \\
& +\frac{1}{\alpha_{2}^{\frac{2}{\beta_{2}}}} \sqrt{\frac{8 \alpha_{2}^{\frac{3}{\beta_{2}}}}{\sqrt{27}} \rho_{\mathrm{b}}} \sum_{i=1}^{n} \mu_{i, \frac{\beta_{2}+1}{2 \beta_{2}}}\left[m, m_{\text {crit }}, \infty\right],
\end{aligned}
$$

such that the generalised effective size is given by the simple equation

$D_{e}=\frac{I_{1}}{I_{2}}$.

The truncated moments of some standard distributions are reported in Jawitz (2004). For lognormal distributions, the truncated moments are given by

$\mu_{k}\left[m, m_{1}, m_{2}\right]=\frac{1}{\sqrt{\pi}} \mu_{k}[m] \cdot \int_{z\left(m_{1}\right)}^{z\left(m_{2}\right)} \exp \left(-z^{2}\right) \mathrm{d} z$

using the transformation

$z=\frac{1}{\sqrt{2}}\left(\frac{\log \left(m / m_{m}\right)}{\log \sigma_{m}}-k \log \sigma_{m}\right)$

leading to expressions involving the error function

$\operatorname{erf}(x):=\frac{2}{\sqrt{\pi}} \int_{0}^{x} \exp \left(-t^{2}\right) \mathrm{d} t$

For evaluating the error function numerically, we use the expression

$\operatorname{erf}(x) \approx 1-\frac{1}{\left(1+x\left(a_{1}+x\left(a_{2}+x\left(a_{3}+x a_{4}\right)\right)\right)\right)^{4}}$

with coefficients

$a_{1}=0.278398, a_{2}=0.230389$,

$a_{3}=0.000972, a_{4}=0.078108$

This approximation leads to an error lower than $\epsilon=5 \cdot 10^{-4}$. 
Acknowledgements. We thank the European Centre for MediumRange Weather Forecasts (ECMWF) for computing time within the framework of the special project SPCHCLAI. We thank the editor and the two anonymous reviewers for helpful comments, which lead to significant improvement of the manuscript.

Edited by: M. Krämer

\section{References}

Chen, T., Rossow, W., and Zhang, Y.: Radiative effects of cloudtype variations, J. Climate, 13, 264-286, 2000.

Clark, T. and Farley, R.: Severe downslope windstorm calculations in two and three spatial dimensions using anelastic interactive grid nesting: A possible mechanism for gustiness, J. Atmos. Sci., 41, 329-350, 1984.

Dean, S., Lawrence, B., Grainger, R., and Heuff, D.: Orographic cloud in a GCM: the missing cirrus, Clim. Dynam., 24, 771-780, 2005.

DeMott, P., Rogers, D., and Kreidenweiss, S.: The susceptibility of ice formation in upper tropospheric clouds to insoluble aerosol components, J. Geophys. Res., 102, 19575-19584, 1997.

DeMott, P., Cziczo, D., Prenni, A., Murphy, D., Kreidenweis, S., Thomsom, D., Borys, R., and Rogers, D.: Measurements of the concentration and composition of nuclei for cirrus formation, $\mathrm{P}$. Nat. Acad. Sci. USA, 100, 14655-14660, 2003.

Ebert, E. and Curry, J.: A parameterization of ice cloud optical properties for climate models, J. Geophys. Res., 97, 3831-3836, 1992.

$\mathrm{Fu}, \mathrm{Q}$. and Liou, K.: Parameterization of the Radiative Properties of Cirrus Clouds, J. Atmos. Sci., 50, 2008-2025, doi:10.1175/15200469(1993)050<2008:POTRPO>2.0.CO;2, 1993.

Fusina, F., Spichtinger, P., and Lohmann, U.: The impact of ice supersaturated regions and thin cirrus on radiation in the mid latitudes, J. Geophys. Res., 112, D24514, doi:10.1029/2007JD008449, 2007.

Gayet, J.-F., Ovarlez, J., Shcherbakov, V., Ström, J., Schumann, U., Minikin, A., Auriol, F., and Petzold, A.: Cirrus cloud microphysical and optical properties at southern and northern midlatitudes during the INCA experiment, J. Geophys. Res., 109, D20206, doi:10.1029/2004JD004803, 2004.

Jawitz, J. W.: Moments of truncated continuous univariate distributions, Adv. Water Resour., 27, 269-281, 2004.

Joos, H., Spichtinger, P., Lohmann, U., Gayet, J.-F., and Minikin, A.: Orographic cirrus in the global climate model ECHAM5, J. Geophys. Res., 113, D18205, doi:10.1029/2007JD009605, 2008.

Joos, H., Spichtinger, P., and Lohmann, U.: Orographic cirrus in a future climate, Atmos. Chem. Phys., 9, 7825-7845, doi:10.5194/acp-9-7825-2009, 2009.

Joos, H., Spichtinger, P., and Lohmann, U.: Influence of a future climate on the microphysical and optical properties of orographic cirrus clouds in ECHAM5, J. Geophys. Res., 115, D19129, doi:10.1029/2010JD013824, 2010.

Kärcher, B. and Lohmann, U.: A parameterization of cirrus cloud formation: Homogeneous freezing of supercooled aerosols, J. Geophys. Res., 107, 4010, doi:10.1029/2001JD000470, 2002.

Kärcher, B. and Lohmann, U.: A parameterization fo cirrus cloud formation: Heterogeneous freezing, J. Geophys. Res., 108, 4402, doi:10.1029/2002JD003220, 2003.
Kärcher, B., Möhler, O., DeMott, P. J., Pechtl, S., and Yu, F.: Insights into the role of soot aerosols in cirrus cloud formation, Atmos. Chem. Phys., 7, 4203-4227, doi:10.5194/acp-7-4203-2007, 2007.

Khvorostyanov, V. and Curry, J.: The Theory of Ice Nucleation by Heterogeneous Freezing of Deliquescent Mixed CCN. Part II: Parcel Model Simulation, J. Atmos. Sci., 62, 261-285, 2005.

Klein, S. and Hartmann, D.: The seasonal Cycle of Low Stratiform Clouds, J. Climate., 6, 1587-1606, 1993.

Koop, T., Luo, B., Tsias, A., and Peter, T.: Water activity as the determinant for homogeneous ice nucleation in aqueous solutions, Nature, 406, 611-614, 2000.

Meerkötter, R., Schumann, U., Doelling, D. R., Minnis, P., Nakajima, T., and Tsushima, Y.: Radiative forcing by contrails, Ann. Geophys., 17, 1080-1094, doi:10.1007/s00585-999-10807, 1999.

Möhler, O., Field, P. R., Connolly, P., Benz, S., Saathoff, H., Schnaiter, M., Wagner, R., Cotton, R., Krämer, M., Mangold, A., and Heymsfield, A. J.: Efficiency of the deposition mode ice nucleation on mineral dust particles, Atmos. Chem. Phys., 6, 30073021, doi:10.5194/acp-6-3007-2006, 2006.

Prusa, J., Smolarkiewicz, P., and Wyszogrodzki, A.: EULAG, a computational model for multiscale flows, Comput. Fluids, 37, 1193-1207, 2008.

Sassen, K. and Benson, S.: Ice nucleation in cirrus clouds: A model study of the homogeneous and heterogeneous modes, Geophys. Res. Lett., 27, 521-524, 2000.

Schiller, C., Krämer, M., Afchine, A., Spelten, N., and Sitnikov, N.: Ice water content of Arctic, midlatitude, and tropical cirrus, J. Geophys. Res., 113, D24208, doi:10.1029/2008JD010342, 2008.

Spichtinger, P. and Cziczo, D.: Impact of heterogeneous ice nuclei on homogeneous freezing events in cirrus clouds, J. Geophys. Res., 115, D14208, doi:10.1029/2009JD012168, 2010.

Spichtinger, P. and Gierens, K. M.: Modelling of cirrus clouds Part 1a: Model description and validation, Atmos. Chem. Phys., 9, 685-706, doi:10.5194/acp-9-685-2009, 2009a.

Spichtinger, P. and Gierens, K. M.: Modelling of cirrus clouds - Part 1b: Structuring cirrus clouds by dynamics, Atmos. Chem. Phys., 9, 707-719, doi:10.5194/acp-9-707-2009, 2009b.

Spichtinger, P. and Gierens, K. M.: Modelling of cirrus clouds - Part 2: Competition of different nucleation mechanisms, Atmos. Chem. Phys., 9, 2319-2334, doi:10.5194/acp-9-2319-2009, 2009c.

Wendisch, M., Yang, P., and Pilewskie, P.: Effects of ice crystal habit on thermal infrared radiative properties and forcing of cirrus, J. Geophys. Res., 112, D08201, doi:10.1029/2006JD007899, 2007.

Wylie, D. and Menzel, W.: Eight Years of High Cloud Statistics Using HIRS, J. Climate., 12, 170-184, 1999.

Zhang, M., Lin, W., Klein, S., Bacmeister, J., Bony, S., Cederwall, R., DelGenio, A., Hack, J., Loeb, N., Lohmann, U., Minnis, P., Musat, I., Pincus, R., Stier, P., Suarez, M., Webb, M., Wu, J., Xie, S., Yao, M., and Zhang, J.: Comparing clouds and their seasonal variations in 10 atmospheric general circulation models with satellite measurements, J. Geophys. Res., 110, d15S02, doi:10.1029/2004JD005021, 2005. 